\title{
TGF- $\beta$-driven muscle degeneration and failed regeneration underlie disease onset in a DMD mouse model
}

Davi A.G. Mázala, ${ }^{1}$ James S. Novak, ${ }^{1,2,3}$ Marshall W. Hogarth, ${ }^{1}$ Marie Nearing, ${ }^{1,4}$ Prabhat Adusumalli, ${ }^{1}$ Christopher B. Tully, ${ }^{1}$ Nayab F. Habib, ${ }^{1}$ Heather Gordish-Dressman, ${ }^{1,2,3}$ Yi-Wen Chen, ${ }^{1,2}$ Jyoti K. Jaiswal, ${ }^{1,2,3}$ and Terence A. Partridge ${ }^{1,2,3}$

'Center for Genetic Medicine Research, Children's Research Institute, Children's National Hospital, Washington, DC, USA. 2Department of Genomics and Precision Medicine and ${ }^{3}$ Department of Pediatrics, The George Washington University School of Medicine and Health Sciences, Washington, DC, USA. ${ }^{4}$ Department of Medicine, University of Alabama at Birmingham, Birmingham, Alabama, USA.

Duchenne muscular dystrophy (DMD) is a chronic muscle disease characterized by poor myogenesis and replacement of muscle by extracellular matrix. Despite the shared genetic basis, severity of these deficits varies among patients. One source of these variations is the genetic modifier that leads to increased TCF- $\beta$ activity. While anti-TCF- $\beta$ therapies are being developed to target muscle fibrosis, their effect on the myogenic deficit is underexplored. Our analysis of in vivo myogenesis in mild (C57BL/10ScSn- $m d x /$ ] and C57BL/6J- $m d x \Delta$ 52) and severe DBA/2J-mdx (D2-mdx) dystrophic models reveals no defects in developmental myogenesis in these mice. However, muscle damage at the onset of disease pathology, or by experimental injury, drives up TGF- $\beta$ activity in the severe, but not in the mild, dystrophic models. Increased TGF- $\beta$ activity is accompanied by increased accumulation of fibroadipogenic progenitors (FAPs) leading to fibro-calcification of muscle, together with failure of regenerative myogenesis. Inhibition of TCF- $\beta$ signaling reduces muscle degeneration by blocking FAP accumulation without rescuing regenerative myogenesis. These findings provide in vivo evidence of early-stage deficit in regenerative myogenesis in D2-mdx mice and implicates TCF- $\beta$ as a major component of a pathogenic positive feedback loop in this model, identifying this feedback loop as a therapeutic target.

Authorship note: DAGM and JSN are co-first authors.

Conflict of interest: The authors have declared that no conflict of interest exists.

Copyright: () 2020, American Society for Clinical Investigation.

Submitted: December 12, 2019

Accepted: February 26, 2020

Published: March 10, 2020.

Reference information: /CI Insight. 2020;5(6):e135703.

https://doi.org/10.1172/jici.

insight.135703.

\section{Introduction}

Duchenne muscular dystrophy (DMD) is a lethal muscle disease caused by mutations that result in the absence of dystrophin protein, a cytoplasmic protein that helps tether the cytoskeletal actin to the extracellular matrix $(1,2)$. DMD affects about $1 / 6200$ boys, who suffer from skeletal muscle wasting and premature death due to cardiorespiratory failure (3). Lack of dystrophin exacerbates myofiber damage during muscle activity, leading to chronic degeneration-regeneration and inflammation of muscles (4). Despite the ability of skeletal muscle to fully regenerate myofibers, patients with DMD show progressive deposition of fibrous extracellular matrix (ECM), which replaces muscle fibers and severely compromises muscle function (5). The degenerative and regenerative deficits contributing to disease progression and underlying muscle pathology in DMD remain to be elucidated $(6,7)$. Failed regeneration and ongoing degeneration observed in DMD muscles implicate dysregulation of muscle-resident myogenic and nonmyogenic stem cells. Interactions between these stem cells and inflammatory cells are crucial to efficient muscle regeneration $(8,9)$. An understanding of how dystrophin deficiency initiates changes in these stem cell functions and interactions is required for rational development of therapies to address these secondary consequences of dystrophin deficiency.

Proteomic and histopathological analysis of DMD mouse models - C57BL/10ScSn- $m d x / \mathrm{J}(\mathrm{B} 10-m d x)$ and C57BL/6J-mdx $\Delta 52$ (B6- $m d x$ ) - reveals a sudden onset of pathological changes at 3-4 weeks of age (10). At this time rapid postnatal myogenesis slows down in healthy mice but accelerates in $m d x$ models (11). B10-mdx and B6- $m d x$ models cope well with myogenic demands at this age (12). However, introduction of the B10-mdx dystrophin mutant allele into the DBA/2J (D2) genetic background (D2-mdx) manifests in a more severe pathology 
accompanied by a myogenic deficit (13-15), placing the D2-mdx model closer in disease severity and myogenic deficit to patients with $\mathrm{DMD}(16,17)$.

The latent transforming growth factor binding protein 4 (LTBP4) gene in the D2 mice carries a polymorphism that removes a 12 -amino acid region that enables the LTBP4 protein to sequester TGF- $\beta$, and failure to do so increases active TGF- $\beta$ levels during muscle inflammation (18). High TGF- $\beta$ activity is a feature of $\operatorname{DMD}(19,20)$. As in the D2- $m d x$ mouse, LTBP4 polymorphisms that increase TGF- $\beta$ activity are associated with increased disease severity in patients with $\operatorname{DMD}(21,22)$. TGF- $\beta$ has been shown to both limit myogenesis and promote fibrosis (23-26), but poor myogenesis itself is also associated with fibrosis (27-29). Thus, it is uncertain whether TGF- $\beta$ directly inhibits early myogenesis and independently drives fibrosis or whether there is some interaction between the 2 activities in D2-mdx mice and in boys with DMD. Myogenesis of skeletal muscle involves complex interactions between myogenic cells and a variety of other muscle-resident cell types (8). In vitro systems lack these interactions and offer insights only into the cell-intrinsic satellite cell deficits. On the other hand, in vivo studies limit experimental access and need to consider the complications associated with disturbed cell populations during late stages of disease. Here, we have performed in vivo studies to investigate the influence of the matrix and other muscle-resident cells on myogenesis at the onset of disease symptoms to avoid such complications.

Fibroadipogenic progenitors (FAPs) are muscle-resident cells that increase transiently during individual episodes of normal muscle repair, facilitating myogenesis and ECM formation, before being cleared by apoptosis (30-32). However, chronic muscle damage and inflammation in muscular dystrophies favor the persistence of FAPs, hindering regeneration and leading to muscle fibrosis, adipogenesis, and osteogenesis (31-33). In response to acute injury, infiltrating macrophages secrete tumor necrosis factor, which promotes FAP survival (34). However, chronic damage and inflammation (as in DMD), as well as other drivers of elevated TGF- $\beta$ activity, inhibit FAP apoptosis, allowing them to differentiate into fibrogenic and other ECM-secreting cells (34).

Here we have performed a quantitative in vivo analysis of satellite cell-mediated myogenesis and FAP-mediated muscle degeneration in the mild (B10-mdx and B6- $m d x$ ) and severe (D2-mdx) mouse models of DMD. We find that the onset of disease is accompanied in the milder B10-mdx and B6-mdx models by a rapid rise in regenerative myogenesis that is lacking in the more severe D2-m $x$ model. This myogenic deficit correlates with the level of active TGF- $\beta$, which is highest in the D2-mdx model. Disease onset in this model also shows prominent accumulation of FAPs with areas of muscle degeneration and fibro-calcified deposits. These regions also exhibit a paucity of satellite cells and lack of regenerating myofibers. In vitro analysis showed that FAPs derived from D2- $m d x$ muscles underwent spontaneous osteogenic differentiation, while FAPs isolated from B10- $m d x$ did not. Experimental injury of the less affected muscles in the D2-mdx model re-created the pathologies observed in highly affected muscles, namely high TGF- $\beta$ level, excessive FAP accumulation, fibro-calcified deposits, and lack of myogenesis. These features mimic deficits reported in muscles from patients with $\mathrm{DMD}$, thus indicating the D2-mdx mouse as a pertinent model in which to investigate the inflammatory and degenerative pathology seen in DMD and to delineate the interplay between the muscle degenerative and regenerative processes that drive myogenic deficit in this disease. Further, using this model, we establish the utility of inhibiting TGF- $\beta$ activity to ameliorate FAP-mediated fibro-calcified muscle degeneration. We show this inhibition alone is not sufficient to reverse the myogenic deficit, identifying the relevance of the D2-mdx model to investigate the complexity of the downstream effects of TGF- $\beta$ hyperactivity and for discovery of combinatorial drugs that can simultaneously restrict muscle degeneration and activate myogenesis in dystrophic muscles.

\section{Results}

Myonuclear accumulation during postnatal growth of DMD mouse models. To evaluate developmental and spontaneous regenerative myogenesis in WT and dystrophic mice, we first examined these processes in vivo using our previous approach of counting the number of nuclei in single isolated extensor digitorum longus (EDL) muscle fibers of the C57BL/6 (B6) WT and $m d x$ (exon 52-null) mice (Supplemental Figure 1, A-F; supplemental material available online with this article; https://doi.org/10.1172/jci. insight.135703DS1) (11). Presenting the number of myonuclei per fiber as normalized cumulative frequency plots (Figure 1, A and B; and Supplemental Figure 1, G and H) shows that each population is distributed as a compact sigmoid curve with median values, $\mathrm{Y}=0.50$, of the myonuclei/fiber corresponding closely to the means. This shows quasinormal distributions such that juvenile growth over the course of 4-14 weeks comprises a coherent rightward shift of each curve with increasing age. This indicates 
A

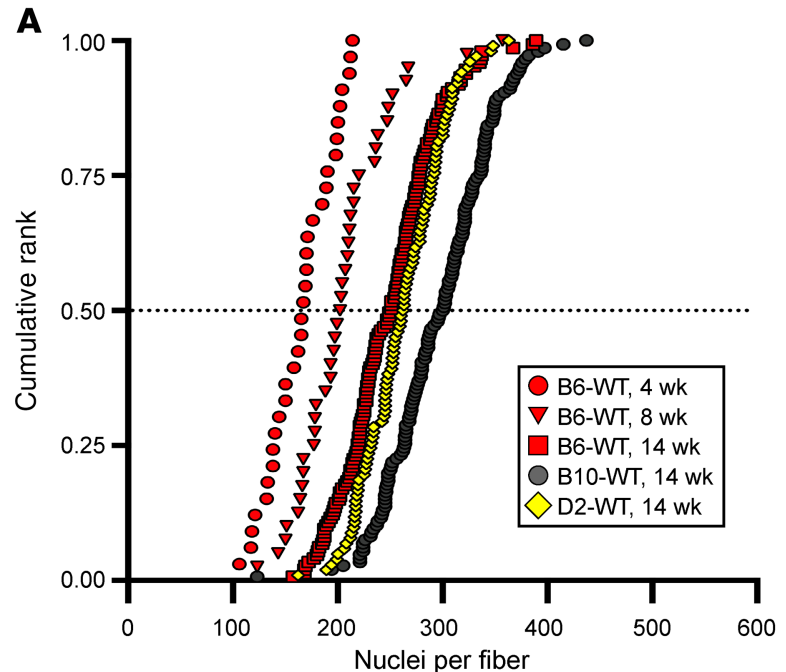

C

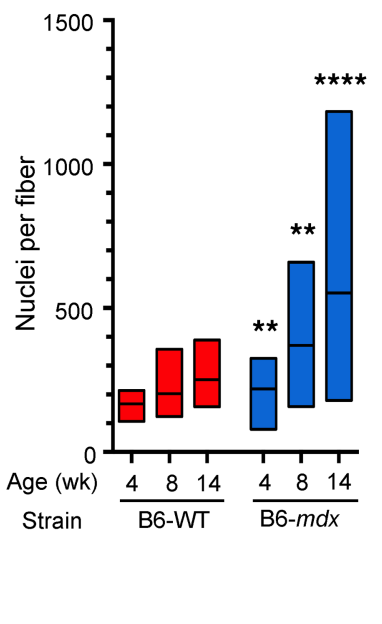

D

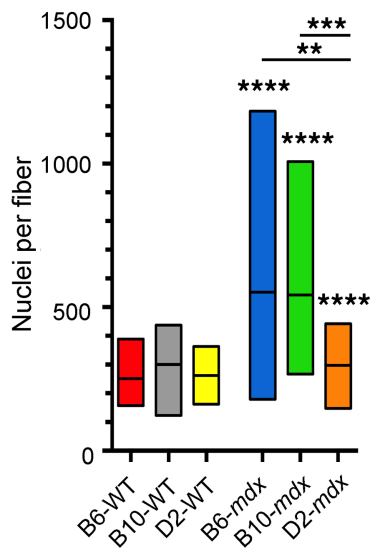

B

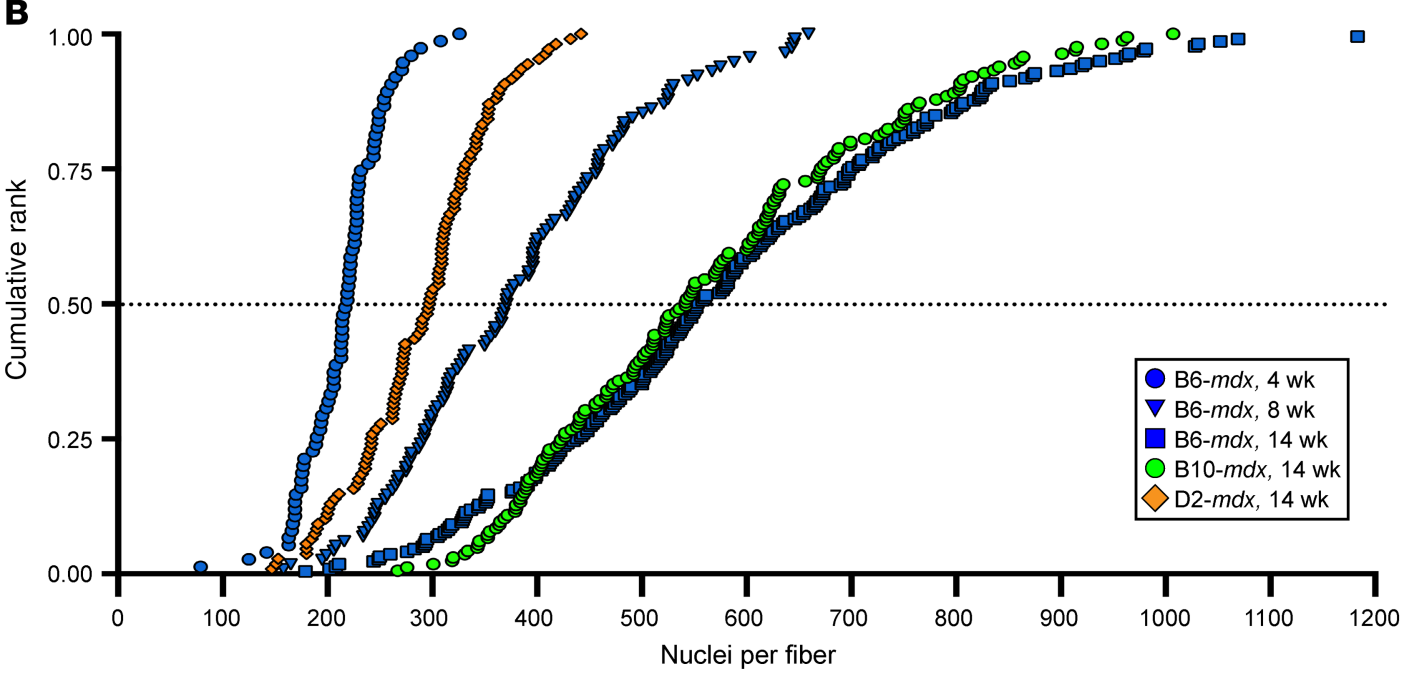

Figure 1. Myonuclear incorporation in EDL single muscle fibers during development in different dystrophic mouse models. (A and B) Cumulative ranks demonstrating the distribution of nuclei per myofiber from WT and dystrophic mice at 4, 8, and 14 weeks of age. (A) Number of nuclei per fiber from 4-week-old B6-WT ( $n=33)$, 8-week-old B6-WT $(n=40)$, 14-week-old B6-WT $(n=147)$, 14-week-old B10-WT $(n=146)$, and 14-week-old D2-WT ( $n=102)$. (B) Number of nuclei per fiber from 4-week-old B6- $m d x(n=75)$, 8-week-old B6- $m d x(n=116), 14$-week-old B6- $m d x(n=218), 14$-week-old B10- $m d x(n=165)$, and 14-week-old D2- $m d x(n=108)$. (C) Box-and-whisker plots showing the median and interquartile range of nuclei per myofiber from B6-WT and B6- $m d x$ at 4 ( $n=33$ for B6-WT; $n=75$ for B6- $m d x), 8$ ( $n=40$ for B6-WT; $n=116$ for B6- $m d x)$, and 14 ( $n=147$ for B6-WT; $n=218$ for B6- $m d x)$ weeks of age. (D) Box-and-whisker plots showing the median and interquartile range of nuclei per myofiber at 14 weeks of age from B6- $m d x$, B10- $m d x$, D2- $m d x$, and their corresponding WT controls. For $\mathbf{C}$ and $\mathbf{D},{ }^{* *} P<0.01$; ${ }^{* *} P<0.001$; ${ }^{* * *} P<0.0001$. For statistical analysis, linear regression models clustered by mouse group with Tukey's post hoc multiple-comparisons adjustment were performed.

progressive and conspicuous myonuclear accumulation via an orderly process in which the change of number of myonuclei/fiber shown along the median line is representative of the population as a whole.

In B6-WT mice the pattern of juvenile growth between 4 and 14 weeks is fundamentally similar to what we have seen in B10-WT mice (Figure 1A) (11). A striking finding is the far greater increase in myonuclear number in B10-mdx and B6- $m d x$, reaching near-identical maxima at 14 weeks (Figure 1, A and B). The myonuclear accumulation from 4 to 14 weeks of age was far more rapid in B6- $m d x$ and B10-mdx dystrophic mice than their respective WT controls (Figure 1, A-D). On average, the B6-WT mice added 9 new nuclei to each myofiber between 4 and 8 weeks of age and an additional 8 new nuclei per fiber between 8 and 14 weeks of age, while the myofibers of the B6- $m d x$ mice accumulated 38 nuclei between 4 and 8 weeks of age and 30 nuclei between 8 and 14 weeks of age, a 4 -fold increase (Figure $1 \mathrm{C}$ and Table 1). Consequently, EDL myofibers of 14-week-old B6-mdx mice contained twice as many myonuclei as those of the B6-WT mice (Figure 1, C and D). Interestingly, this population profile is indistinguishable from that of B10-mdx mice at this same age (Figure 1, B and D). Thus, total myonuclear accumulation is almost identical in these 
Table 1. Rate of myonuclear incorporation in young B6-WT and B6-mdx myofibers

\begin{tabular}{|c|c|c|c|c|c|c|}
\hline \multirow{2}{*}{$\begin{array}{l}\text { Strain } \\
\text { Age (weeks) }\end{array}$} & \multicolumn{3}{|c|}{ B6-WT } & \multicolumn{3}{|c|}{ B6-mdx } \\
\hline & 4 & 8 & 14 & 4 & 8 & 14 \\
\hline$n$ value (fibers) & 33 & 40 & 147 & 75 & 116 & 218 \\
\hline Median (nuclei/fiber) & 167 & 202 & 251 & 219 & 371 & 552 \\
\hline $\begin{array}{l}\text { Nuclear incorporation } \\
\text { (nuclei/fiber/week) }\end{array}$ & 4-8 weeks: 8.9/week & & 8.1/week & $4-8$ we & & 8-14 weeks: 31.2/week \\
\hline
\end{tabular}

Analysis of the data shown in Figure 1, showing the mean and median nuclei per fiber, as well as the rate of myonuclear incorporation (nuclei/fiber/week) from 4 weeks till 14 weeks of age in both the B6-WT and B6-mdx mouse models.

2 mild models of DMD despite their different genetic backgrounds and dystrophin mutant alleles. In contrast, the mutant allele found in the B10- $m d x$ background associates with more severe pathology on the D2 genetic background $(11,13)$, in which myonuclear accumulation was $50 \%$ lower in 14-week-old D2-mdx myofibers than in those of B6- $m d x$ and B10-mdx mice, corresponding more closely to levels observed in the 4- to 8-week-old B6-mdx myofibers (Figure 1, B and D, and Supplemental Figure 1G). In D2-WT mice myonuclear accumulation at 14 weeks was indistinguishable from that of B6-WT (Figure 1, A and D). These data span the early phase of disease (starting at 4 weeks of age, when the muscles in all of these dystrophic mouse models undergo massive spontaneous degeneration. In the milder models, degeneration was counteracted by a powerful regenerative response, while the severe D2-mdx model showed little regeneration and concomitantly greater disease severity right from the onset of disease.

Early-disease stage muscle histopathology of mild and severe dystrophic muscle. We noted that D2-mdx mouse muscles exhibited severe alterations at the macroscopic level as early as 3-4 weeks of age, where the triceps and pectoral muscles showed clearly visible whitened muscle bundles along their length, which continued to worsen even beyond 5 weeks of age (Figure 2A). In contrast, such gross abnormalities were not detected in any of the age-matched B10-mdx mice (Figure 2A). Based on our previous observations of heightened fibrosis and inflammation in the D2-mdx model (13), we first examined collagen deposition in muscles at this early disease stage. We monitored the presence of fibrous collagen in triceps of 38-day-old D2- $m d x$ mice using Masson's trichrome stain in conjunction with second harmonic generation microscopy (Figure 2, B and C). Both approaches clearly identified deposition of fibrous collagen within the fascicles of D2-mdx muscles (Figure 2, B and C). Additionally, using alizarin red staining, we determined that the macroscopic muscle whitening in D2-mdx mice was due to muscle degeneration where myofibers were lost to calcification (Figure 2D). This was not seen at 14 days of age but became apparent at 21 days of age in D2-mdx muscle, but not in B10-mdx muscles of the same age (Figure 2, D and E). From 21 to 38 days of age, the areas of calcification increased cumulatively in D2-mdx muscles, doubling from under $5 \%$ at 21 days to covering over $10 \%$ of the entire muscle cross-sectional area by 38 days (Figure 2F; and Supplemental Figure 2). In contrast, B10-mdx muscles showed relatively small regions of myonecrosis without the presence of calcification even at 38 days of age compared with D2-mdx (Figure 2, E and F; and Supplemental Figure 2). Interestingly, by 38 days of age, myofiber regeneration, as indicated by the presence of CNFs, was over 3-fold higher in B10- $m d x$ ( $>40 \%$ of all fibers) as compared with approximately $12 \%$ in D2- $m d x$ (Figure $2 \mathrm{G}$ ). Thus, at the onset of spontaneous muscle degeneration at approximately 3 weeks of age, the D2-mdx muscle is not accompanied by the vigorous regeneration seen in the B10-mdx mouse, and necrotic spaces are replaced instead by patchy fibrosis and extensive calcification.

Failed myogenesis and FAP osteogenesis mark spontaneous muscle degeneration in young D2-mdx mice. To determine whether the low frequency of CNFs in D2-mdx muscles are indicative of selective loss of regenerating myofibers or a failure of satellite cell function, we first evaluated the expression of myogenic transcriptional factors, i.e., paired box 7 (Pax7), myoblast determination protein 1 (MyoD), and myogenin, at 2 ages around the onset of pathogenesis in the mild (B10-mdx) and severe (D2- $m d x$ ) models (Figure 3, A-C). Although there were no significant differences in gene expression at 21 days of age between these models, expression of $\mathrm{Pax} 7, \mathrm{MyoD}$, and myogenin were all significantly lower in $\mathrm{D} 2-m d x$ muscles at 38 days of age than in B10-mdx muscles (Figure 3, A-C). 
A

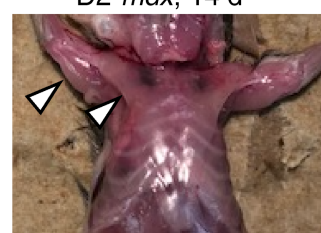

B

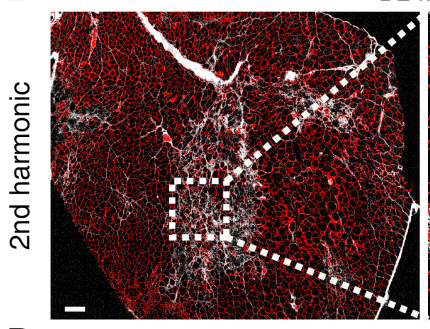

D
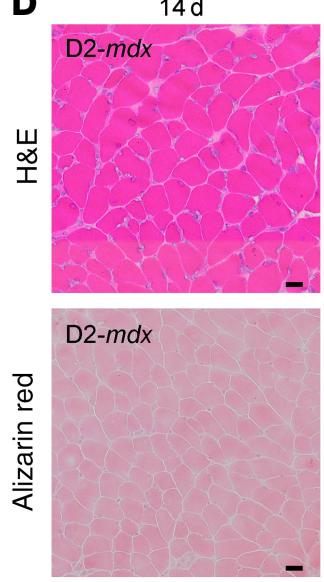

E

$14 d$
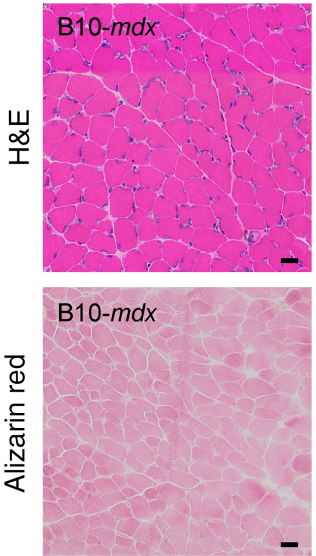

$D 2-m d x, 21 d$

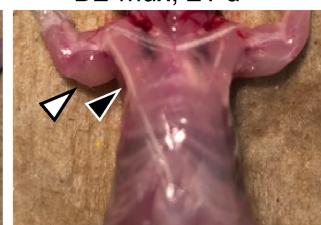

$\mathrm{D} 2-m d x$
D2- $m d x, 38 d$

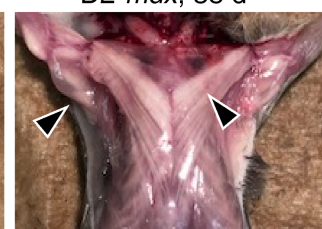

C
D2-WT, $38 d$

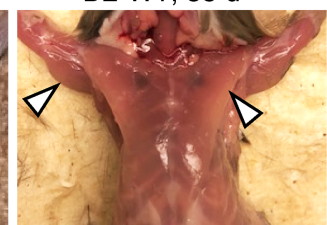

$\mathrm{D} 2-m d x$
B10- $m d x, 38 d$
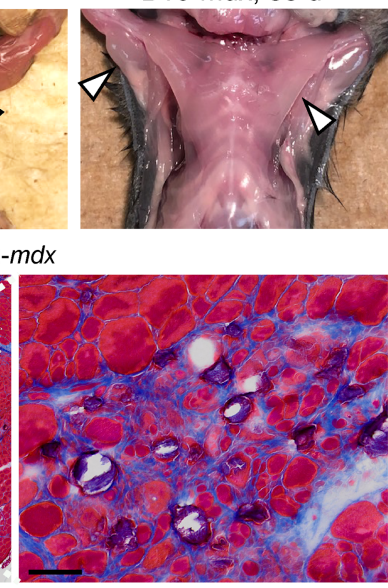

$\mathbf{F}$

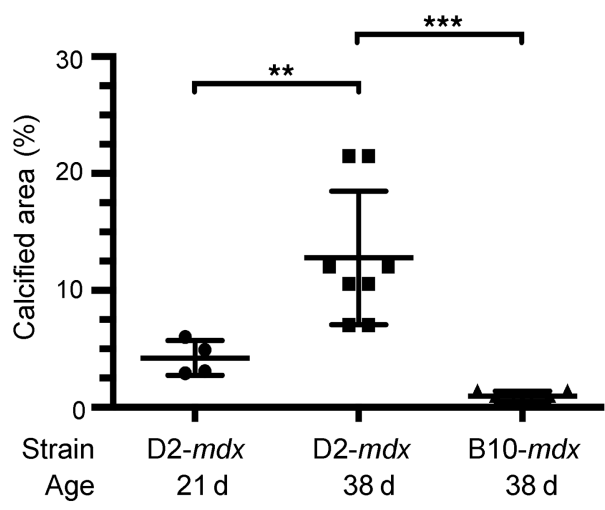

G

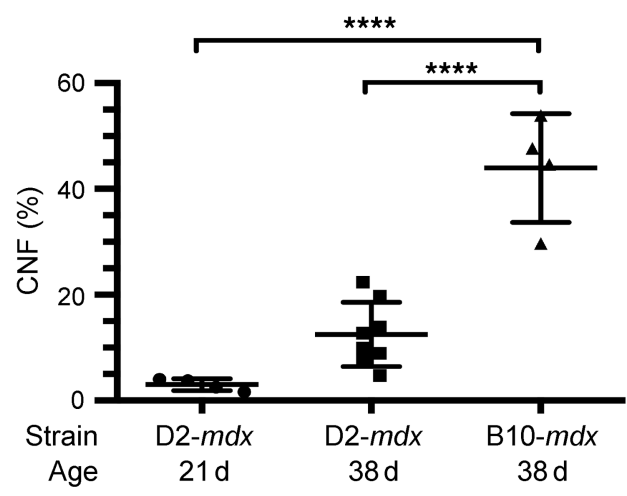

Figure 2. Muscles from D2-mdx mice show evidence of muscle degeneration and calcification starting at the onset of disease. (A) Macroscopic images of 14-, 21-, and 38-day-old D2-mdx mice, 38-day-old D2-WT mice, and 38-day-old B10-mdx mice. D2-mdx mice show development of conspicuous pale stripes in the pectoral muscles between 21 and 38 days of age accompanied by similar changes in other muscles (e.g., triceps, black arrowhead) corresponding to areas of calcification identified in sections by alizarin red staining (D). No abnormal changes were seen in either D2-WT or B10-mdx mice (white arrowhead). (B and C) Signs of endomysial fibrosis in and around areas of degeneration in the triceps muscle were identified by second harmonic generation (B) and trichrome stain (C) in muscles of D2-mdx mice at 38 days of age. (D and E) H\&E and alizarin red staining of triceps muscle sections from D2-mdx and B10-mdx mice at 14, 21, and 38 days of age. (F and G) Quantification of the percentage of calcified area and centrally nucleated fibers (CNFs) in triceps muscles from B10-mdx and D2-mdx at 21 days and 38 days old. (F) Quantification of percentage of calcification from 21-day-old D2-mdx mice ( $n=4)$, 38-day-old D2-mdx mice $(n=7)$, and 38-day-old B10-mdx mice $(n=4)$. (G) Quantification of CNF percentage from 21-day-old D2-mdx mice $(n=4)$, 38-day-old D2-mdx mice $(n=8)$, and 38-day-old B10-mdx mice $(n=4)$. For F and $\mathbf{G}$, data are expressed as mean \pm SD. One-way ANOVA with Tukey's post hoc comparison. ${ }^{* *} P<0.01 ;{ }^{* *} P<0.001 ;{ }^{* * *} P<0.0001$. Scale bars: $50 \mu \mathrm{m}$ (B and C), $20 \mu \mathrm{m}$ (D and E). 
A
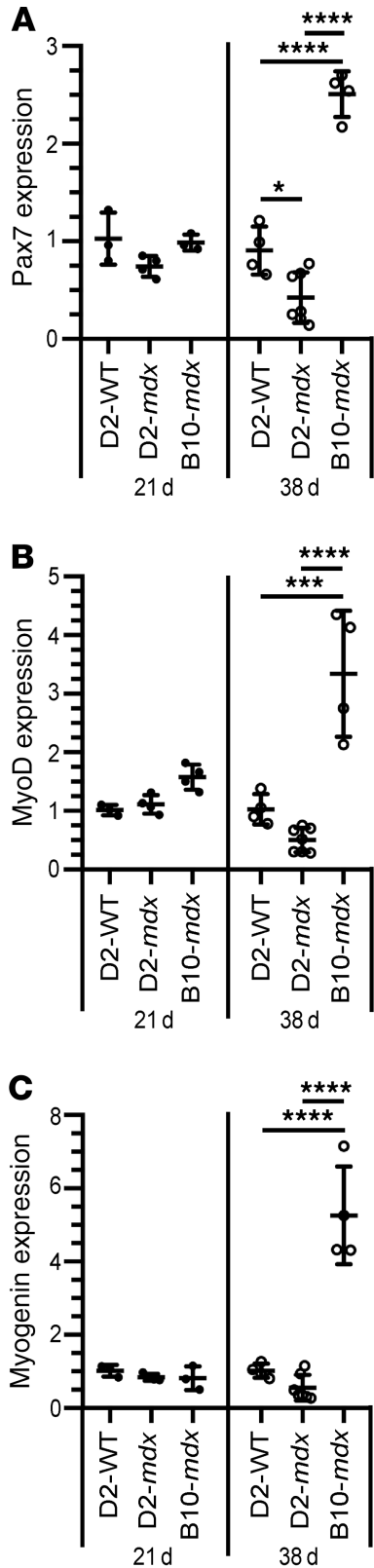

D

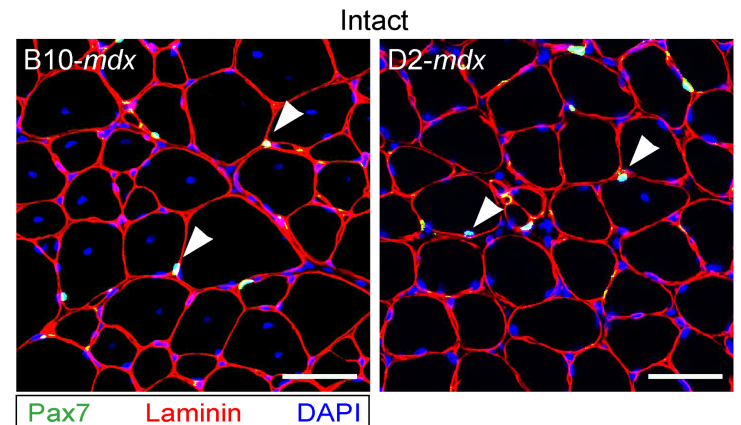

F

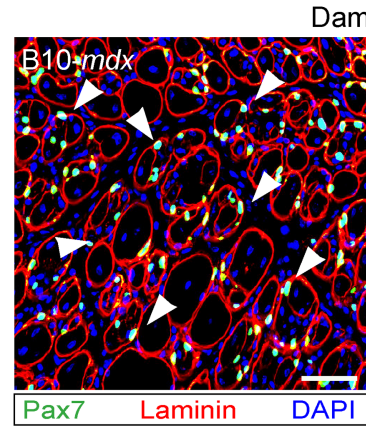

E
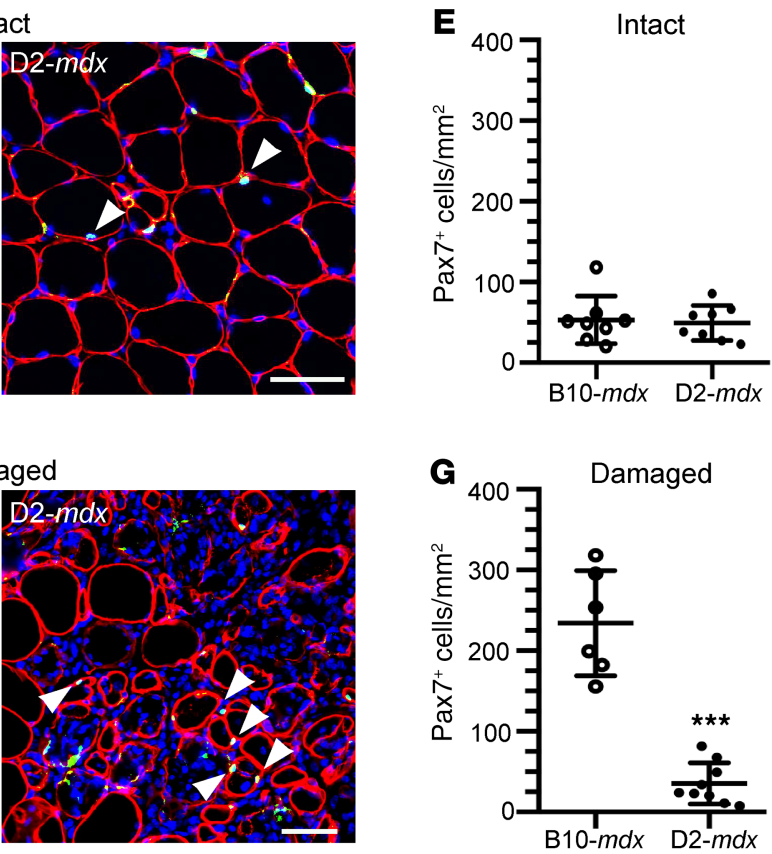

G

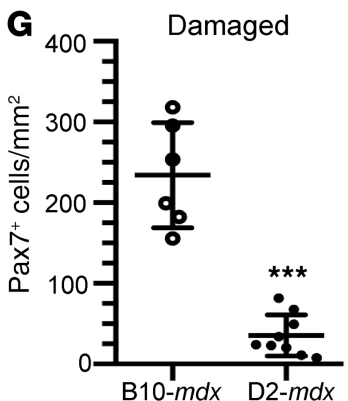

H BrdU labeling period 3d Labeling . ...........Euthanize at 38d old 6d Labeling 9d Labeling
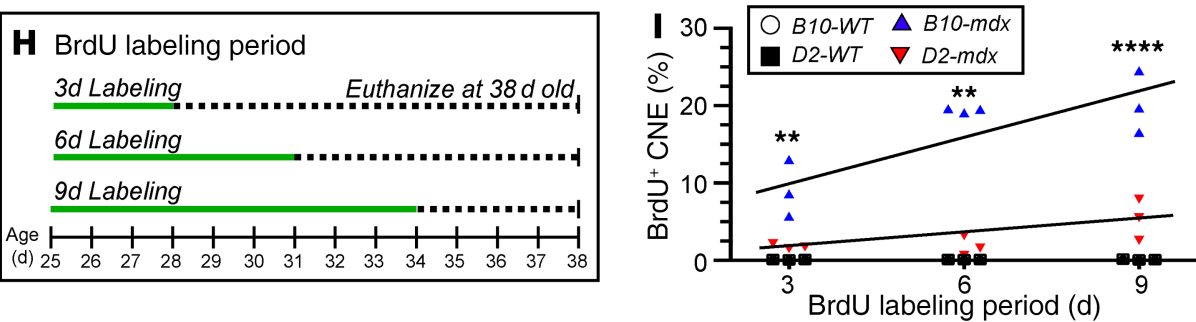

\section{J}
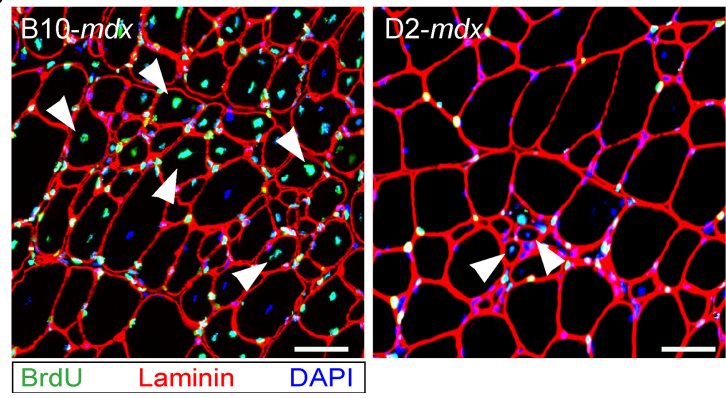

$\mathbf{K}$

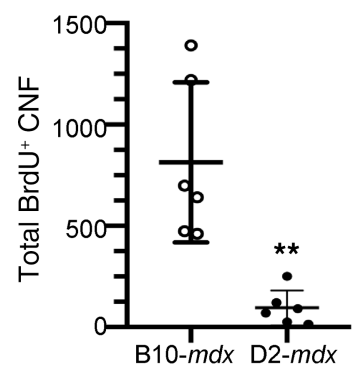

Figure 3. Satellite cells from D2-mdx mice show limited myogenic capacity during spontaneous muscle regeneration. (A-C) Gene expression of Pax7, MyoD, and myogenin from 21- and 38-day-old D2-WT ( $n=3$ per age), D2- $m d x(n=4$ per age), and B10- $m d x(n=3$ per age) mice. (D) Immunofluorescence (IF) images stained for labeling Pax7 $7^{+}$satellite cells in intact areas of the triceps muscle from 38-day-old B10-mdx and D2-mdx mice. White arrowheads, Pax $7^{+}$cells. (E)

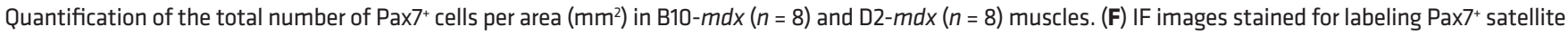
cells in damaged areas of the triceps muscle from 38-day-old B10- $m d x$ and D2-mdx mice. White arrowheads, Pax7 $7^{+}$cells. (G) Quantification of the total number of Pax $7^{+}$cells per area $\left(\mathrm{mm}^{2}\right)$ in muscles from B10- $m d x(n=6)$ and D2- $m d x(n=9)$. (H) Schematic figure demonstrating the pattern for pulsed 5'-bromo-2'-deoxyuridine (BrdU) labeling of proliferating satellite cells. (I) Quantification of BrdU+ CNFs relative to total fibers per muscle section ( $n=3$ for each labeling period per genotype). (J) IF images from the triceps muscle from 38-day-old B10-mdx and D2-mdx mice labeled with BrdU for 9 days. White arrowheads, BrdU+ CNFs. (K) Quantification of the total number of BrdU+ CNFs after 6-9 days of BrdU labeling in muscle sections from 38-day-old B10- $m d x(n=6)$ and D2- $m d x(n=6)$ mice. For $\mathbf{A}, \mathbf{B}$, and $\mathbf{C},{ }^{*} P<0.05$, ${ }^{* *} P<0.001$, and ${ }^{* * * *} P<0.000$; 1-way ANOVA with Tukey's post hoc comparison; for $\mathbf{E}, \mathbf{G}, \mathbf{I}$, and $\mathbf{K},{ }^{* *} P<0.01$, ${ }^{* *} P<0.001$, and ${ }^{* * * *} P<0.0001$; 2-tailed, nonparametric Mann-Whitney $U$ test. Scale bars: $50 \mu \mathrm{m}$ (D, F, and J).

Simple visual inspection of muscle cross sections showed a clear difference between 38-day-old B10$m d x$ and D2-mdx mice regarding the way that areas of muscle fiber damage were resolved. In B10-mdx mice there was clear evidence of rapid myofiber regeneration with a high incidence of small CNFs with occasional inflammatory cells and damaged myofibers (Figure 2D, Supplemental Figure 2A). In contrast, 
areas of damage in D2- $m d x$ muscles did not resolve in this way and remained largely devoid of regenerated myofibers, the space being occupied by inflammatory cells, acellular debris, and calcified areas between the muscle fibers (Figure 2E, Supplemental Figure 2B). The areas in each muscle that were free of inflammatory cells, damaged myofibers, and small-caliber CNFs were classified as "intact" while areas characterized by the presence of 1 or more of these features were classified as "damaged" (Figure 3). In both strains, the damaged areas were readily distinguished from intact areas because the latter contained closely packed polygonal fibers, with peripherally placed myonuclei and few inflammatory cells (Figure 3). Intact muscle areas contained similar numbers of $\mathrm{Pax}^{+}$cells in B10- $m d x$ and D2- $m d x$ (Figure 3, D and E), while damaged areas contained 7-fold fewer Pax $7^{+}$cells in D2- $m d x$ than B10-mdx muscles (Figure 3, E and G).

To further analyze satellite cell function in vivo, we used the thymidine analog BrdU to label recently generated myofiber nuclei. We labeled proliferating myogenic cells by administering BrdU for 3, 6, or 9 consecutive days starting at 25 days of age and counted those myofibers that contained a BrdU-labeled central nucleus as an indicator of the frequency of myoblasts that had proliferated during that labeling period and subsequently fused into regenerating myofibers (Figure 3, H and I). B10-mdx mice in the 9-day labeled group displayed a progressive increase in the proportion of BrdU-labeled fibers, reaching up to $20 \%$ of the total fibers. In contrast, only $5 \%$ of total fibers in D2-mdx muscles were labeled with BrdU after the same 9-day labeling period (Figure 3I). Quantification of BrdU-labeled CNFs indicated 6-fold more muscle regeneration in the $\mathrm{B} 10-m d x$ than in $\mathrm{D} 2-m d x$ muscles (Figure 3, $\mathrm{J}$ and $\mathrm{K}$ ). Collectively, these data indicate that the phenotypic differences between the 2 models at the onset of disease are largely attributable to substantial muscle damage in both but with a markedly limited regeneration in D2-mdx mice.

Myonecrotic muscle diseases, such as DMD and limb girdle muscle dystrophy 2B, feature chronic overactivation of FAPs, marked by platelet-derived growth factor receptor- $\alpha$ (PDGFR $\alpha$ ), which contributes to disease pathology by promoting fibrosis and adipogenesis $(35,36)$. We thus investigated the involvement of these mechanisms in the prominent muscle degeneration in D2-mdx muscle. Immunostaining revealed FAPs in intact and spontaneously damaged areas of triceps muscles in both B10-mdx and D2-mdx mice (Figure 4, A and B; and Supplemental Figure 3). However, FAP-containing areas in D2-mdx mice were more extensive than in B10-mdx mice (Figure 4, A-C; and Supplemental Figure 3).

The conspicuous calcified degeneration in D2- $m d x$ and the known role of FAPs in heterotopic ossification of muscles (37) prompted us to investigate whether increased disease severity in the D2-mdx FAPs was associated with pathways favoring ossification, indicated by increased alkaline phosphatase (ALP) activity. We found that primary PDGFR $\alpha^{+}$FAPs isolated from the 28-day-old D2-mdx muscle underwent extensive spontaneous osteogenic differentiation in vitro (Figure 4, D and E), marked by positive staining for ALP. In contrast to FAPs isolated from D2- $m d x$, FAPs from B10- $m d x$ showed extremely low levels of spontaneous osteogenesis, suggesting that a difference in the cellular niche drives FAP proliferation and adoption of an osteogenic fate as early as 28 days of age. Bone morphogenetic protein 2 (BMP2) has been shown to induce FAP osteogenesis (37). Thus, next we examined D2-mdx FAPs for enhanced sensitivity for osteogenic differentiation by BMP2. Again, D2- $m d x$ FAPs showed increased osteogenesis in response to BMP2 induction, while B10-mdx FAPs were unresponsive to osteogenic differentiation even in response to BMP2 stimulation (Figure 4, F and G). Together, the above results indicate that the severe muscle pathology in young D2-mdx mice is attributable to a combination of conspicuous muscle degeneration and failed regenerative response of satellite cells in the context of increased FAP accumulation and osteogenesis.

Injury triggers poor regeneration and excessive degeneration of muscle of D2-mdx mice. Our observation of limited regenerative capacity in D2- $m d x$ muscles in response to spontaneous damage (Figure 3) prompted us to examine whether the deficit in regenerative competence is a more generalized feature of satellite cells in this model. Accordingly, we induced muscle damage in D2-mdx mice, choosing the tibialis anterior (TA) muscle, in which spontaneous damage is limited at this age. Following a single injection of the myotoxin notexin (NTX), at 24 days of age, we monitored myogenic response by BrdU labeling on successive single days, starting 1day postinjury and continuing daily up to 6 days postinjury. Animals were euthanized 3 days after the end of BrdU administration to allow full nuclear incorporation into myofibers and help delineate the timing and extent of satellite cell proliferation responsible for regeneration of these damaged muscles. Just 1 day following NTX injury (i.e., 1-day BrdU labeling period; Figure 5, A and B), over 30\% of fibers contained a BrdU ${ }^{+}$myonucleus in the NTX-injured B10- $m d x$ mice, while only $6 \%$ of total fibers were BrdU $\mathrm{U}^{+}$ in NTX-injured D2-mdx muscles (Figure 5B). In muscles receiving BrdU at 2 days postinjury, $13 \%$ of fibers were $\mathrm{BrdU}^{+}$in $\mathrm{B} 10-m d x$ muscles, while only $5 \%$ of $\mathrm{D} 2-m d x$ fibers were $\mathrm{BrdU}^{+}$. At the 3 -day time point, the 
A
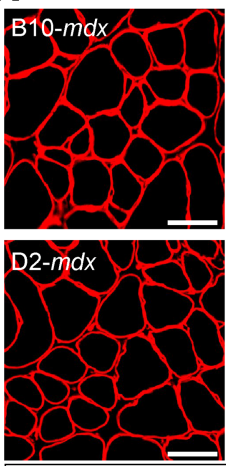

Laminin
Intact
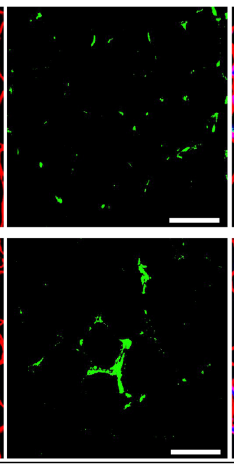

PDGFR
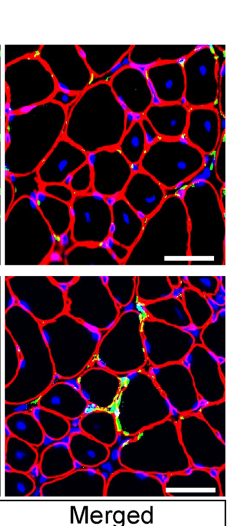

B
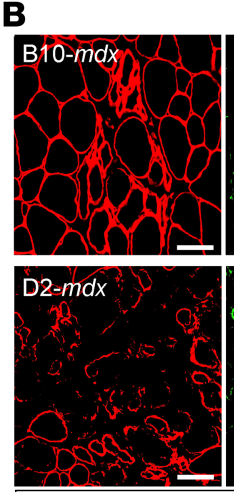

Laminin
Damaged
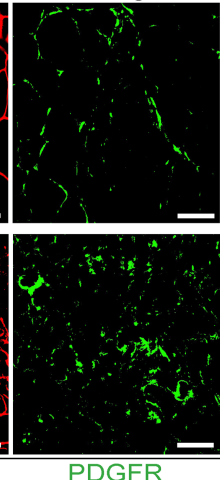
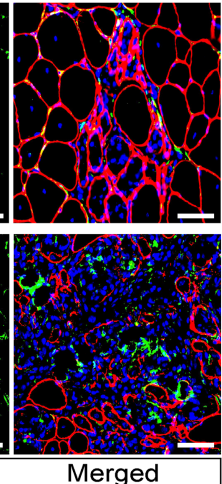

C

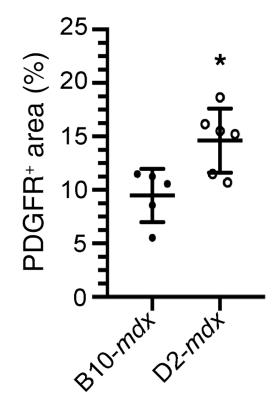

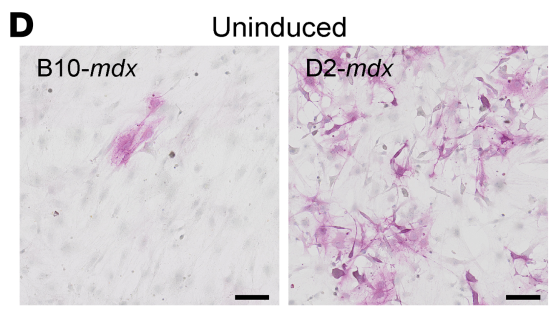

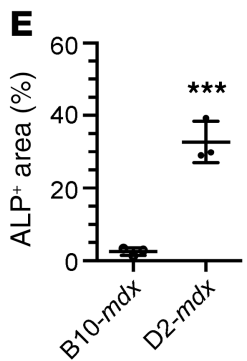

F

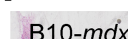

BMP2 induced

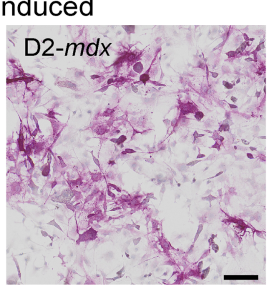

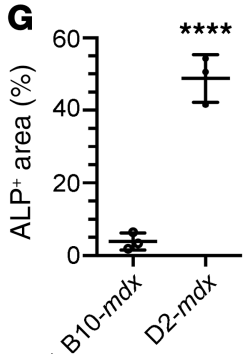

Figure 4. Increased FAP proliferation is responsible for early calcification in muscles from D2-mdx mice. (A and B) IF images stained for PDCFR $\alpha^{+}$ cells in intact (A) and damaged (B) areas in triceps from 38-day-old B10- $m d x$ and D2- $m d x$ mice. Note the higher accumulation of PDCFR $\alpha^{+}$cells in muscles from D2-mdx mice, showing increased presence of FAPs. (C) Quantification of PDGFR $\alpha^{+}$areas is shown as a percentage of the total triceps muscle section area from B10- $m d x(n=5)$ and D2- $m d x$ mice $(n=6)$. (D) Alkaline phosphatase (ALP) staining of primary FAPs isolated from B10-mdx and D2- $m d x$ muscle after 11 days in culture. (E) Quantification of ALP+ area as a measure of osteogenic differentiation of B10- $m d x$ and D2- $m d x$ FAPs ( $n=3$ replicates/genotype). (F and $\mathbf{G}$ ) Images and quantification of in vitro FAP osteogenesis following stimulation with $25 \mathrm{ng} / \mathrm{mL}$ recombinant BMP2. For C, ${ }^{*} P<0.05$; 2-tailed, nonparametric Mann-Whitney $U$ test. For E and $\mathbf{G},{ }^{* *} P<0.001 ;{ }^{* * *} P<0.0001 ; 2$-way ANOVA, Holm-Šídák's multiple-comparisons test. Scale bars: $50 \mu \mathrm{m}$ (A and $\mathbf{B}), 100 \mu \mathrm{m}$ (D and $\mathbf{F})$.

level of labeling in NTX-injured D2- $m d x$ muscles was indistinguishable from the contralateral muscle, where it probably reflects the low background regeneration of spontaneous dystrophic lesions. On subsequent days, BrdU incorporation had returned to the baseline levels seen in noninjured contralateral muscles in both models. These data indicate that the decreased BrdU incorporation in D2-mdx muscle was not the result of a simple delay in myogenesis but a deficit in satellite cell expansion and fusion in D2-mdx muscles following injury, which is consistent with our results for spontaneous damage by the disease process.

A count of $\mathrm{Pax}^{+}$cells was conducted to determine whether the limited myoblast fusion observed following NTX injury of D2-mdx muscle was accompanied by a reduction in the number of satellite cells. The numbers of $\mathrm{Pax}^{+}$cells were nearly 2 -fold lower in D2-mdx muscles than in B10-mdx muscles (Figure $5, \mathrm{C}$ and $\mathrm{D})$, mirroring the satellite cell proliferation and fusion deficit observed in spontaneously degenerating D2- $m d x$ muscles (Figure 3, E and G). To determine whether there was any association between the limited myogenesis in NTX-injured D2- $m d x$ muscles and the amount of muscle damage, we counted CNFs and measured both calcification and total degeneration per cross section of NTX-injured TA muscles (Figure 5, E-G). The percentage of CNFs was 3-fold lower in NTX-injured D2-mdx muscles than in B10- $m d x$ muscles (Figure 5E). Further, the degenerated areas that were calcified were 10 -fold greater (Figure $5 \mathrm{~F}$ ) while the total degenerated areas were 3-fold greater in D2-mdx as compared with B10-mdx (Figure $5 \mathrm{G}$ ). These findings indicate that the lower regenerative myogenesis and greater degeneration in the D2-mdx muscle occur independent of the cause of muscle damage and are inherent deficits in the myogenic maintenance mechanisms of the D2-mdx muscle.

Our earlier observation of FAP expansion in response to spontaneous damage (Figure 4, A-C) prompted us to investigate the underlying degenerative process after NTX injury (Figure 5, H-J; and Supplemental Figure 4). NTX was administered intramuscularly into the TAs of both B10- $m d x$ and D2-mdx, and FAPs were assessed by PDGFR $\alpha$ labeling. At 14 days postinjury, the percentages of muscle area occupied by PDGFR $\alpha^{+}$cells were 1.5-fold higher in D2- $m d x$ than in B10- $m d x$ muscles (Figure $5 \mathrm{H}$ ). This increase was 
A

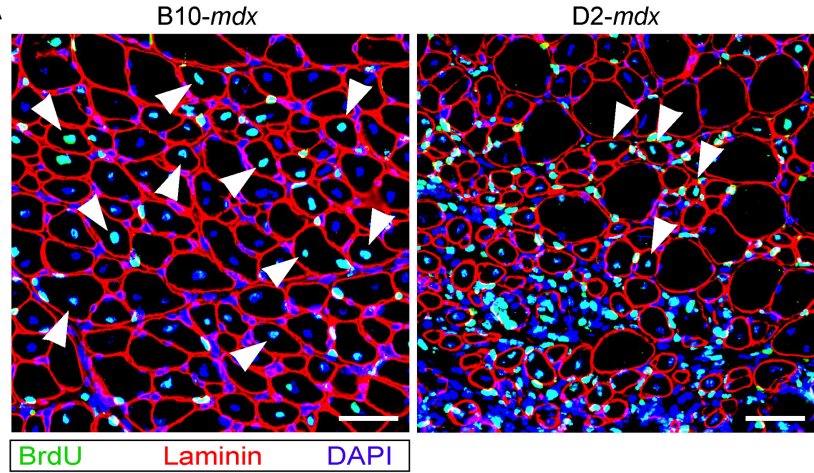

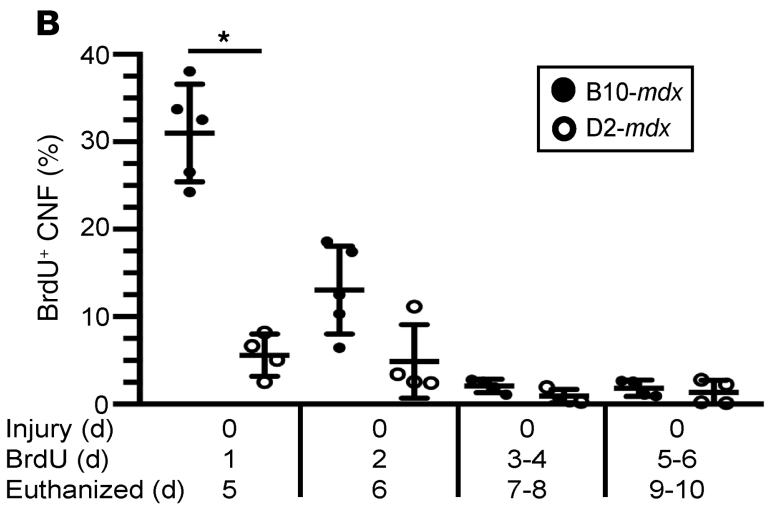

D

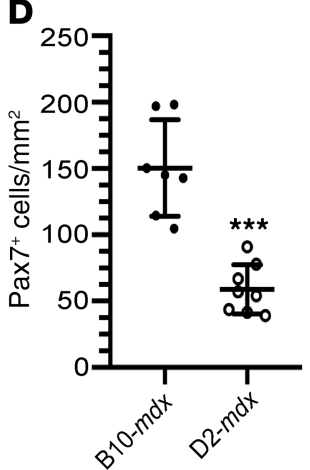

E

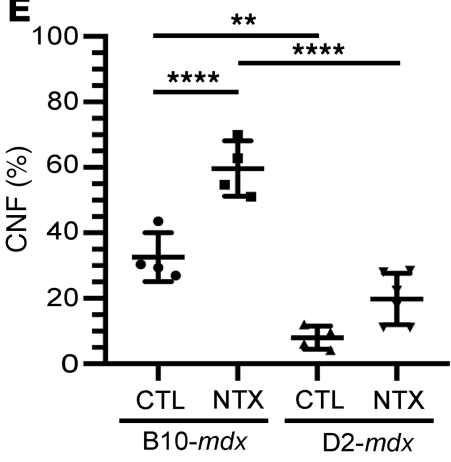

H

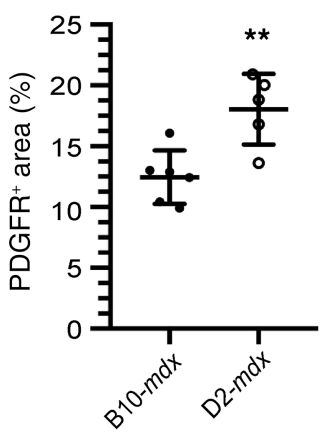

I

F
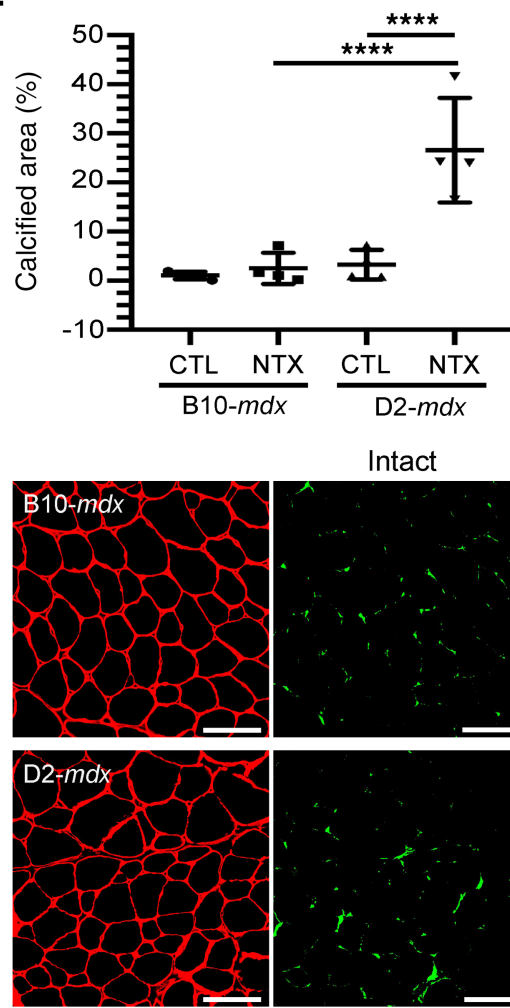

Laminin
Intact
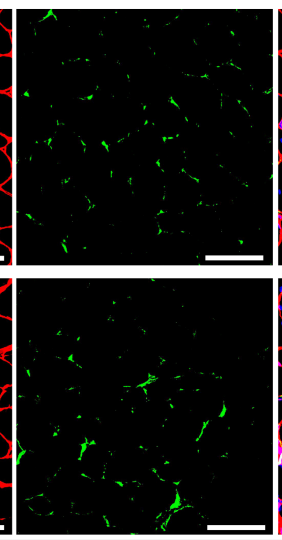

PDGFR

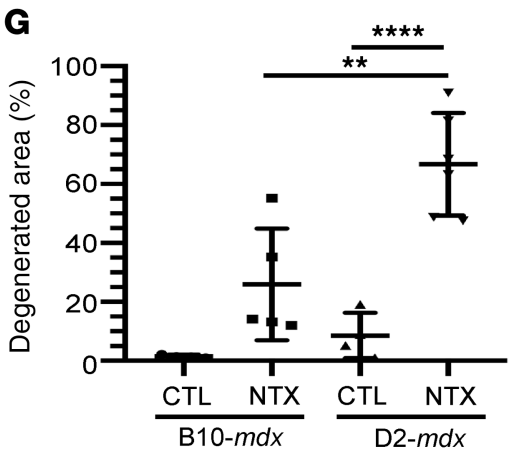

$J$
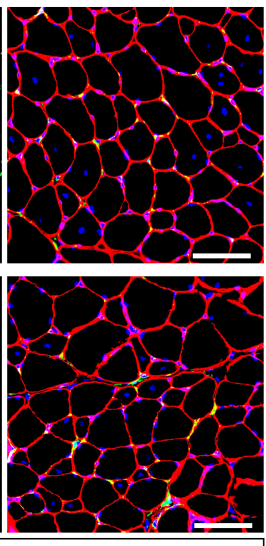

Merged
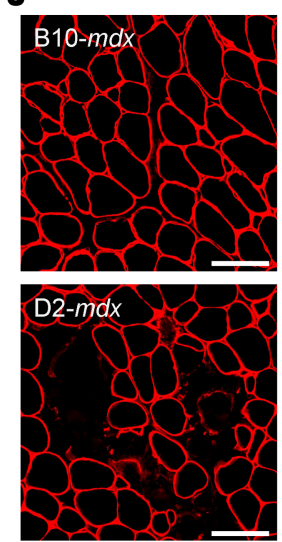

Laminin
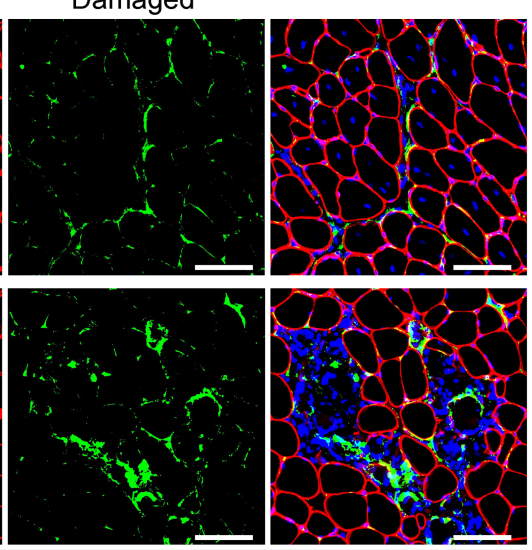

PDGFR

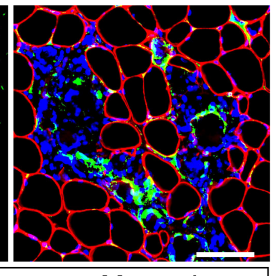

Merged

Figure 5. Staggered BrdU labeling after synchronized NTX-induced injury in TA muscles from B10-mdx and D2-mdx mice. (A) IF images from B10-mdx and D2-mdx NTX-injected TAs labeled with BrdU from 24 to 48 hours postinjury (day 1 labeling). White arrowheads, BrdU+ CNFs. (B) Percentage of BrdU ${ }^{+}$ CNFs across the 6-day labeling period after NTX-injury: B10- $m d x(n=5$ for day $1 ; n=5$ for day 2; $n=4$ for days 3 and $4 ; n=4$ for days 5 and 6 post-BrdU) and D2- $m d x\left(n=4\right.$ for day $1 ; n=4$ for day $2 ; n=4$ for days 3 and $4 ; n=4$ for days 5 and 6 post-BrdU). (C) IF images stained for labeling Pax $7^{+}$cells in NTX-injured TAs from B10- $m d x$ and D2- $m d x$ mice. White arrowheads, Pax7 $7^{+}$cells. (D) Quantification of the total number of Pax $7^{+}$cells per area ( $m m^{2}$ ) at $5-6$ days postinjury in NTX-injured TAs from B10- $m d x(n=7)$ and D2- $m d x(n=8)$. (E-G) Percentage of CNFs, calcification, and total area of degeneration from B10-mdx 
( $n=4$ for CTL, and $n=5$ for NTX TAs) and D2- $m d x$ ( $n=4$ for CTL, and $n=5$ for NTX TAs). (H-J) Quantification and IF images for PDGFR $\alpha^{+}$cells 14 days after NTX injury of TA muscles from B10- $m d x$ and D2-mdx mice (injury was performed at 24 days of age). (H) Quantification of PDCFR $\alpha^{+}$areas shown as a percentage of total muscle section from B10- $m d x(n=6)$ and D2- $m d x$ mice $(n=5)$. For $\mathbf{B}$ and $\mathbf{D}-\mathbf{H},{ }^{*} P<0.05$, ${ }^{* *} P<0.01$, and ${ }^{* * *} P<0.001 ; 2$-tailed, nonparametric Mann-Whitney $U$ test $(\mathbf{B}, \mathbf{D}$, and $\mathbf{H}) ;{ }^{* *} P<0.01$, and ${ }^{* * *} P<0.0001 ; 1$-way ANOVA with Tukey's post hoc comparison (E-G). Scale bars: $50 \mu \mathrm{m}$.

even greater in the NTX-damaged regions than in adjacent undamaged regions (Figure 5, I and J; and Supplemental Figure 4). D2-mdx NTX-injured muscles showed strong and persistent PDGFR $\alpha$ staining within the inflamed interstitium that remained in a state of active inflammation even at 14 days postinjury (Figure 5J and Supplemental Figure 4). This implicates dysregulation of FAPs in D2-mdx muscle as a source of prolonged state of degeneration and perhaps accentuation of the myogenic deficit in this model.

Decreased TGF- $\beta$ activity attenuates FAP-mediated muscle degeneration in D2-mdx muscles. Because high TGF- $\beta$ activity reduces FAP accumulation following muscle injury and also inhibits satellite cell function in vitro (23-26), we hypothesized that increased TGF- $\beta$ activity due to LTBP4 mutation in D2- $m d x$ mice may contribute to disease pathogenesis in D2- $m d x$ mice. We found that at 21 days of age, the time point for the first signs of spontaneous muscle damage in D2- $m d x$ mice, there was no significant increase in the levels of active TGF- $\beta$ (Figure 6A). However, in the following 2 weeks, the levels of active TGF- $\beta$ in D2- $m d x$ muscles increased by 6- and 10-fold of that seen in B10-mdx and D2-WT muscles, respectively (Figure 6B). This increase in active TGF- $\beta$ between 21 and 38 days of age is concordant with an ineffectual myogenesis, and FAP-mediated conspicuous calcification, closely linking the rise in TGF- $\beta$ activity over this period with the overall progression of pathology in this severe $m d x$ model (Figure $6 \mathrm{~A}$ ).

Next, we evaluated the increase in active TGF- $\beta$ levels in response to NTX injury in TA muscles from B10-mdx and D2- $m d x$, at 5 to 6 days postinjury (Figure 6C and Supplemental Figure 5, A and B). NTX injury caused an increase in active TGF- $\beta$ levels by 48 -fold in TA muscles from D2- $m d x$ mice as compared with similarly injured B10-mdx mice (Figure 6C). Thus, heightened TGF- $\beta$ activity correlated with poor myogenesis and excessive FAP-mediated muscle degeneration during both spontaneous and induced injury in D2- $m d x$ mice. To further evaluate the utility of targeting increased TGF- $\beta$ activity between 21 and 38 days of age in slowing disease pathogenesis, we used a drug to inhibit TGF- $\beta$ signaling during this period and assessed myogenesis, degeneration, and overall muscle pathology in D2-mdx muscles. For this, we gave intramuscular (IM) injections of ITD-1, a drug that inhibits TGF- $\beta$ signaling by targeting the TGF- $\beta$ membrane receptor for proteasomal degradation (38). The drug was administered 3 times over 1 week, with the first dose being administered at the time of NTX-induced muscle injury (Figure 6D). BrdU and Pax7 immunostaining were used to systematically monitor the effect of the drug on myogenesis and muscle degeneration. Immunostaining of phosphorylated SMAD3 (p-SMAD30 in situ showed that ITD-1 successfully reduced TGF- $\beta$ activity in the muscles (Figure 6, E and F). This inhibition of TGF- $\beta$ activity in the D2- $m d x$ mice reduced the extent and distribution of active muscle loss indicated by fiber degeneration and infiltration by inflammatory cells in muscles injected with NTX plus ITD-1 (55\% in NTX only; $26 \%$ in NTX + ITD-1 muscles) of total cross-sectional area (Figure 6, I and L). However, it did not increase the number of Pax7-labeled muscle stem cells (Figure 6, G and H) or the number of regenerated myofibers (Figure 6, I-K). On the other hand, it did reduce accumulation of FAPs as indicated by the reduced PDGFR $\alpha$ staining in the treated muscles (Figure 6, M and N), explaining the reduction in muscle calcification and fibrosis (Figure 6, O-R; and Supplemental Figure 5, C and D).

Overall, the above results point to the importance of raised TGF- $\beta$ signaling in increased pathology of D2- $m d x$ muscle and indicate additional pathways that may contribute to myogenic deficit associated with the enhanced disease severity in this mouse model.

\section{Discussion}

Although the primary pathological consequences of lack of dystrophin are death of muscle fibers and chronic inflammation, failure to regenerate lost fibers and their replacement by ECM are major compounding factors in the relentless progression of DMD. Whether the regenerative failure is attributable to degradation of the muscle microenvironment or to the exhaustion of muscle-resident satellite cells is a matter of active debate $(16,17)$. Although deficits are more pronounced in older DMD patients, even satellite cells from young DMD patients exhibit myogenic deficits $(16,17)$. Whether the proliferative failure arises from repeated episodes of regeneration that render the satellite cells intrinsically functionally deficient or whether it is driven by degradation of the ECM, which makes the environment inimical to 
A

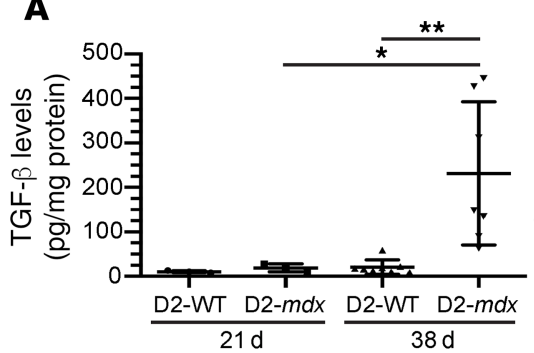

E NTX
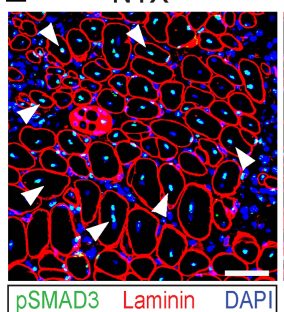

I

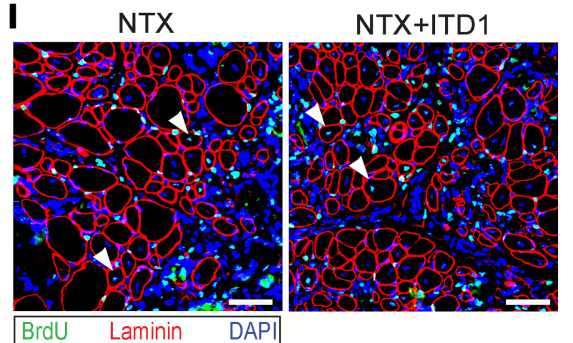

M
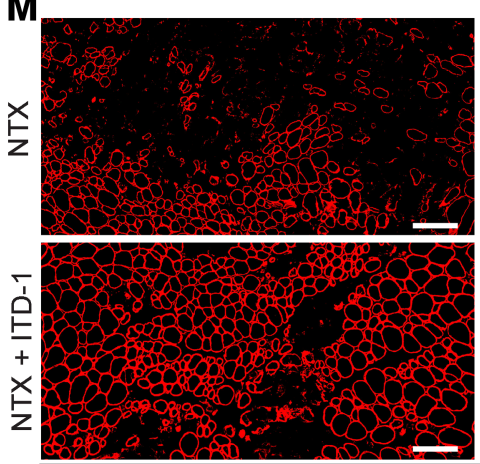

Laminin
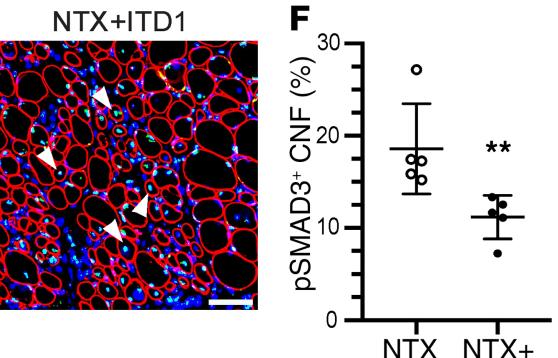

G
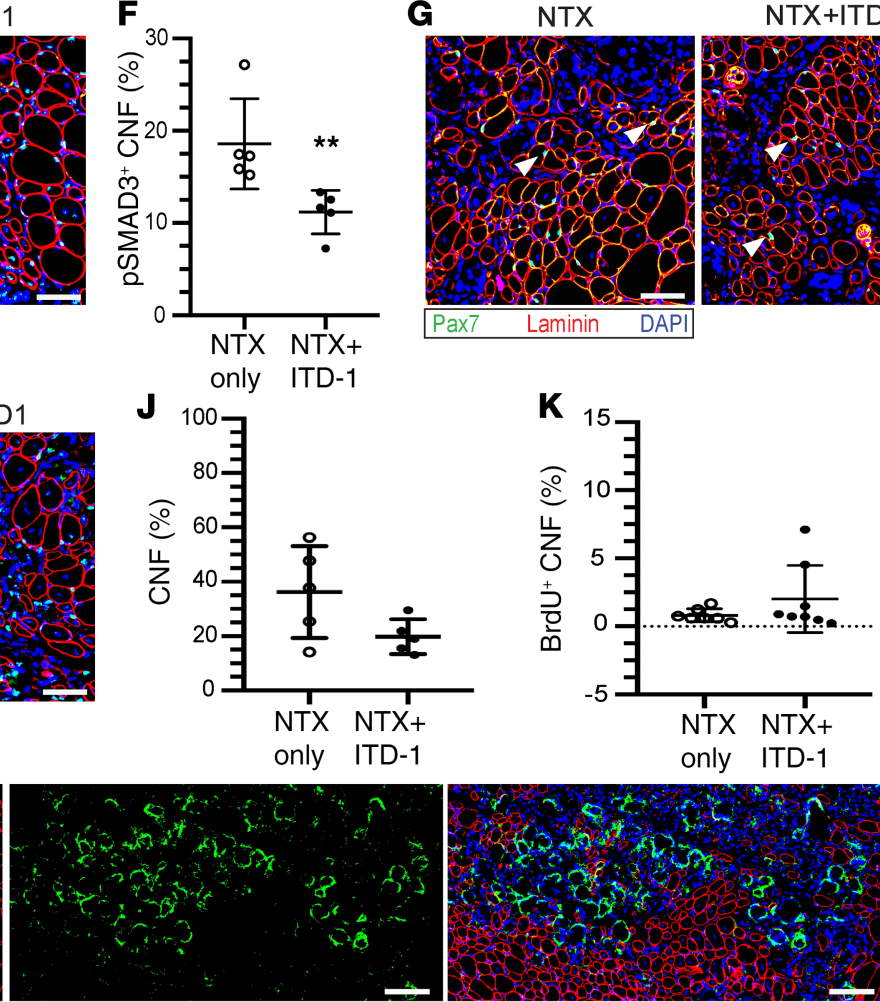

$\mathbf{N}$

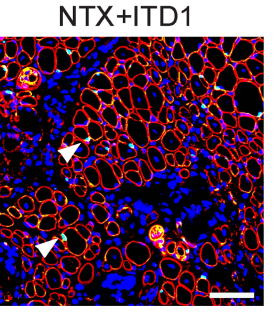

C D NTX $_{\text {N }}$

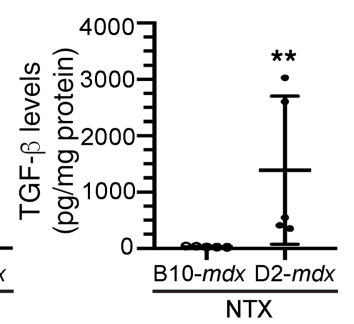

ITD-1

ITD-1

$\frac{2 \mathrm{~d} B r d U}{\operatorname{TTD}-1}$ ITD-1

Animal

dissection

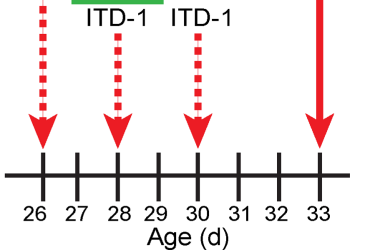

H
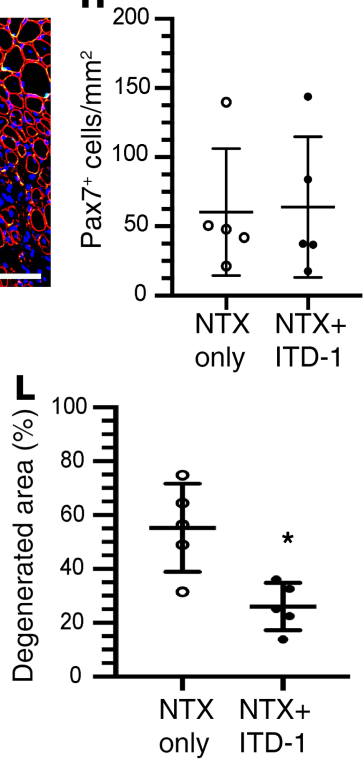
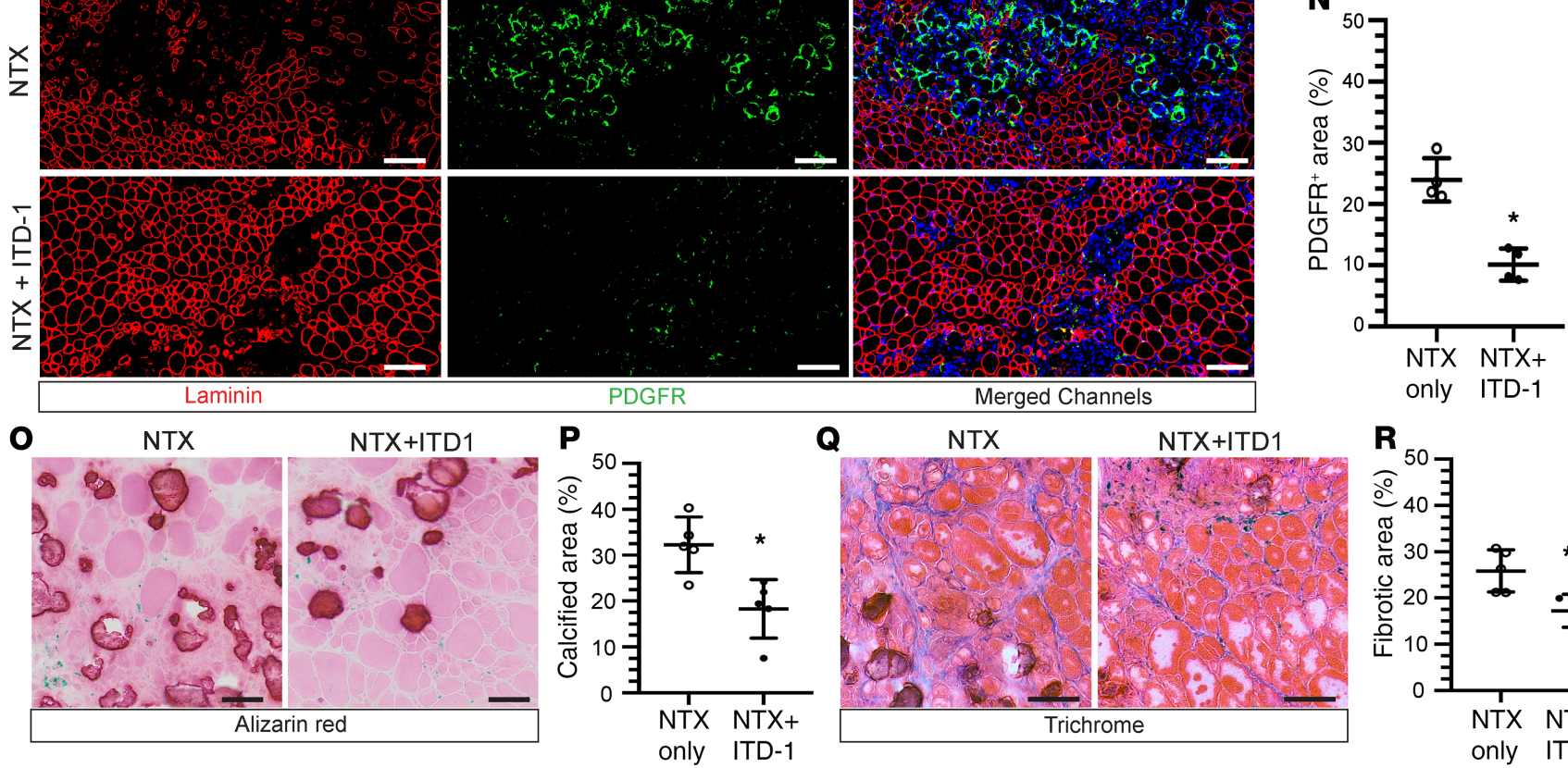

Q

NTX
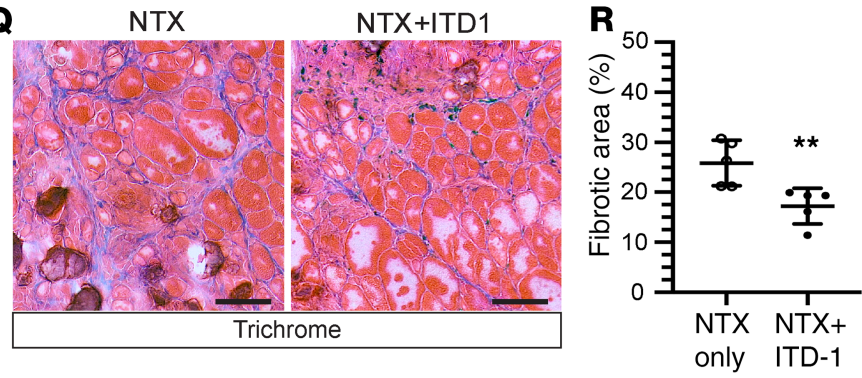

Figure 6. Inhibition of TGF- $\boldsymbol{\beta}$ activity attenuates degeneration and fibro-calcification in D2-mdx muscle. (A and B) Active levels of TGF- $\beta$ in muscle homogenates from 21-day-old D2-WT $(n=3)$ and D2- $m d x(n=3)$, 38-day-old D2-WT $(n=8)$ and D2- $m d x(n=7)$, and 38-day-old B10-WT ( $n=5)$, D2-WT ( $n=$ 8), B10- $m d x(n=4)$, and D2- $m d x(n=7)$ mice. (C) Active levels of TCF- $\beta$ in muscle homogenates from NTX-injured TAs (injury at 24 days of age) from B10$m d x(n=5)$ and D2-mdx $(n=5)$ mice at 5 and 6 days postinjury. (D) TA muscles from 26-day-old D2-mdx mice received an IM injection of NTX alone or NTX plus ITD-1. At 2 and 4 days postinjury, muscles were injected with either saline or ITD-1 (BrdU administered between 24 and 72 hours postinjury). (E and F) IF images and quantification of p-SMAD3+ CNFs in muscles injured with $(n=5)$ or without ITD-1 $(n=5)$. Arrowheads, p-SMAD3 $3^{+}$cells. (G and $\left.\mathbf{H}\right)$ IF images and quantification of total number of Pax $7^{+}$cells per area in muscles injured with $(n=5)$ or without ITD-1 ( $n=5$ ). Arrowheads, Pax $7^{+}$cells. (I-K) IF images and percentage of CNFs and BrdU ${ }^{+}$CNFs in muscles injured with $(n=5)$ or without ITD- $1(n=5)$. Arrowheads, BrdU ${ }^{+}$cells. (L) Quantification of the total area of degeneration relative to total muscle cross section in muscles injured with $(n=5)$ or without ITD-1 ( $n=5$ ). (M and $\mathbf{N}$ ) IF images and quantification 
of PDCFR $\alpha^{+}$areas per muscle area in muscles injured with $(n=4)$ or without ITD- $1(n=4)$. (O-R) Alizarin red and trichrome staining and quantification of muscles injured with $(n=5)$ or without ITD-1 $(n=5)$. For $\mathbf{A}$ and $\mathbf{B},{ }^{*} P<0.05$, ${ }^{* *} P<0.01$, and ${ }^{* * *} P<0.001$; 1 -way ANOVA with Tukey's post hoc comparison; for $\mathbf{C}, \mathbf{F}, \mathbf{L}, \mathbf{N}, \mathbf{P}$, and $\mathbf{R},{ }^{*} P<0.05,{ }^{*} P<0.01$; 2-tailed, nonparametric Mann-Whitney $U$ test. Scale bars: $50 \mu \mathrm{m}(\mathbf{E}, \mathbf{G}, \mathbf{I}, \mathbf{0}$, and $\mathbf{Q}), 100 \mu \mathrm{m}$ (M).

myogenesis, has yet to be resolved. Because the B10- $m d x$ mouse does not reproduce the myogenic deficit of DMD muscle (11), the underlying mechanism may not be directly related to the absence of dystrophin. Here, we show that young D2-mdx mice exhibit a pattern of degradation of the IM environment in association with muscle damage together with failed myogenesis that resembles the picture in DMD, making these young D2-mdx mice a suitable model for evaluation of potential therapeutic avenues to address the mechanisms leading to DMD muscle loss.

Our initial evaluation of histopathological changes in muscles from young D2-mdx mice was triggered by the macroscopic observation that, despite lack of histological abnormalities in muscles of 14-day-old mice, by 21 days these mice showed whitening of some of their muscles. Such changes progressed and were more conspicuous by 38 days of age in D2-mdx but not in the age-matched D2-WT or B10-mdx mice. We found that, in addition to fibrosis, muscle calcification featured heavily in the macroscopic degeneration of these muscles. This is the first report that shows and characterizes the onset of muscle degeneration in D2- $m d x$ mice at 3 weeks of age completely synchronously with calcification. It adds to previous observations of calcification in muscles from 7-week-old D2-mdx mice (13-15) in conjunction with fewer regenerated myofibers than the B10-mdx. This shows that independent of the age, the myogenic deficit goes hand in hand with muscle degeneration.

Developmental and regenerative myogenesis both proceed by activation, proliferation, differentiation, and eventual fusion of muscle-resident satellite cells $(8,39)$. However, our analysis shows no developmental myogenic defect in D2-mdx mice up to the point of emergence of conspicuous muscle degeneration at 21 days in concert with a clear failure of regenerative myogenesis. This led us to investigate whether the mechanism of muscle regeneration itself is altered. We found that expression of myogenic transcription and regulatory factors is markedly lower in the regenerating muscles from D2- $m d x$ muscles than in B10- $m d x$. Likewise, Pax7 staining and incorporation of BrdU-labeled myonuclei demonstrate that proliferation and fusion of myogenic cells are also grossly defective in D2-mdx compared with B10- $m d x$ muscles over this period. Spontaneous regeneration in response to diseaseinduced myofiber death in B10- $m d x$ mice attests to efficient proliferation and fusion of these satellite cells that amounts to the replacement of some $20 \%$ of muscle fibers over a 9 -day period and results in the doubling of myonuclear numbers per myofiber. In contrast, over this same period, myonuclear labeling in the D2-mdx muscle fibers is 4 -fold lower, reaching only up to $5 \%$ of total muscle fibers. Furthermore, our single-day BrdU labeling scheme after a timed NTX injury fully recapitulates poor regenerative myogenesis in the $\mathrm{D} 2-m d x$ muscle.

The discovery that the D2 genetic background reduces the effectiveness of muscle regeneration (13) has stimulated interest in the mechanisms behind the disturbance of this process. An especial focus is the fact that the major primary genetic defect implicated in the enhanced pathology, the LPTB4 mutation, leads to modification of levels of active TGF- $\beta$, a systemic signaling protein implicated in a number of pathological pathways (40). TGF- $\beta$ is a key cytokine that promotes fibroblast proliferation and deposition of fibrotic tissue within the muscle interstitium during dystrophic pathogenesis. However, TGF- $\beta$ is also a central factor in satellite cell activation and function during normal tissue repair, and its decrease during the regenerative phase has been implicated in the apoptotic clearance of FAPs $(26,40-44)$. Here we demonstrated that IM levels of active TGF- $\beta$ rise rapidly between 21 and 38 days of age in D2-mdx mice and that this correlates with the severity of muscle degeneration and limited myogenesis observed over this period in this mouse model. Levels of active TGF- $\beta$ are dramatically higher in D2- $m d x$ muscle than in B10- $m d x$ muscle during spontaneous damage and after NTX-induced injury, implicating both TGF- $\beta$-mediated myogenic deficit and FAP-mediated degeneration in the greater pathology of the D2-mdx muscle.

Aberrations in the proliferation or clearance of FAPs are reported both to be detrimental to regeneration and to contribute to chronic muscle degeneration in myopathies $(31-33,37)$. Chronic muscle damage and inflammation as in DMD are associated with high TGF- $\beta$ activity, leading to suggestions of its role in excess FAP accumulation and pathological ECM deposition (34). Here we report that severely affected D2- $m d x$ muscles, characterized by degeneration, calcification, and initiation of fibrosis, contained more FAPs than B10- $m d x$ muscles, and FAPs have recently been proposed as a driving 
force behind progressive ossification of skeletal muscle (37). Our in vitro work shows that D2-mdx muscle-derived FAPs show autonomous preference for the osteogenic pathway, suggesting an intermediary role for FAPs in calcification in D2- $m d x$ muscles. This contrasts with our findings in B10- $m d x$, where FAP accumulation was a relatively minor feature and osteogenesis was minimal, suggesting that the DBA background has a pathogenic effect on the FAP niche. Because FAPs facilitate myofiber regeneration after injury (44), these findings are consistent with our hypothesis that pathogenic FAP signaling underpins the poor myogenesis we observed in D2-mdx muscle.

More recently, a crosstalk between FAPs and TGF- $\beta$ signaling has been proposed, in which FAP numbers correlate with both TGF- $\beta$ levels and deposition of ECM during tissue regeneration (45). We found that inhibiting TGF- $\beta$ activity in injured muscles of D2- $m d x$ mice, by targeted degradation of the TGF- $\beta$ receptor, directly improved muscle histopathology by limiting the extent of muscle degeneration, calcification, and fibrosis. This implies that the milder muscle phenotype in B10-mdx and B6-mdx is a consequence of decreased FAP accumulation, as has been indicated previously (46). Here, the D2-mdx mouse is shown as a useful model in which to unravel the complex issues of positive feedback between muscle fiber pathology and the acute inflammatory response, in which the FAP population appears to act as a fulcrum. Despite this critical role of FAPs, it is clear from our studies that merely lowering TGF- $\beta$ activity is not sufficient to reverse the myogenic deficit in the D2- $m d x$, thus identifying additional mechanisms to be targeted to fully reverse the myogenic deficit in DMD. While D2-mdx lends itself as a valuable model for identifying and testing such therapeutic approaches, it also gives the prospect of evaluating these more long-term consequences of aberrant TGF- $\beta$ signaling for the sustenance of a chronic metastable state that manifests as unresolving chronic disease. In addition, D2- $m d x$ mice are a valuable model in which to evaluate the effects of the interplay between the individual elements of chronic inflammatory signaling, increased TGF- $\beta$ activity, and abnormal FAP accumulation on the structure of the intercellular matrix, satellite cell function, and muscle regeneration in DMD.

\section{Methods}

Animals. All mice were kept in the same room (typical ambient conditions, $20.9 \% \mathrm{O}_{2}$ and $22 \pm 1{ }^{\circ} \mathrm{C}$ ) and had the same access to food, water, and bedding and light cycle (12-hour light/12-hour dark). Animals were euthanized by cervical dislocation, and tissues were dissected, frozen, and stored at $-80^{\circ} \mathrm{C}$. We used dystrophic mouse models harboring a point mutation in Dmd exon 23, C57BL/10ScSn-mdx/J (B10$m d x)$ and DBA/2J- $m d x(\mathrm{D} 2-m d x)$ mice, and a dystrophic mouse model harboring a deletion of Dmd exon 52 , C57BL/6J-mdx 452 (B6-mdx), as well as their corresponding genotype controls - C57BL/10ScSnJ (B10-WT), DBA/2J (D2-WT), and C57BL/6J (B6-WT). Mice were originally obtained from The Jackson Laboratory, bred in-house, and used for all experiments. Breeding pairs of B6- $m d x$ mice were previously obtained from Shin'ichi Takeda of the National Center of Neurology and Psychiatry in Tokyo, Japan (47).

Myofiber isolation, staining, and imaging. EDL muscle fibers were isolated following our previously described protocol $(48,49)$. EDL muscle was carefully dissected and incubated in $0.2 \%$ collagenase type I (Sigma-Aldrich, C0130) for approximately 2 hours. After collagenase digestion, single fibers were isolated by gentle trituration in DMEM (Lonza, 12-604F), transferred to 2-mL Eppendorf vials containing $30 \%$ sucrose (Sigma-Aldrich, S0389), and stored at $-80^{\circ} \mathrm{C}$. On the day of staining, Eppendorf vials were thawed at $4^{\circ} \mathrm{C}$, and sucrose solution was removed and replaced with blocking buffer $(0.5 \%$ Triton X-100, $0.1 \%$ Tween $20,2 \%$ BSA, and $20 \%$ goat serum in TBS-Tween buffer) and incubated at $4^{\circ} \mathrm{C}$ overnight. After blocking, fibers were washed and resuspended in/with Alexa Fluor 594-conjugated phalloidin for 20 minutes and left overnight at $4^{\circ} \mathrm{C}$ in TBS with Tween buffer. On the following day fibers were mounted with ProLong Gold Antifade with DAPI (P36931, Life Technologies).

BrdU labeling. BrdU (Sigma-Aldrich, B9285) was administered ad libitum in sterile drinking water at a concentration of $0.8 \mathrm{mg} / \mathrm{mL}$ and kept protected from light during administration (50). BrdU was administered from 25 to 28 days ( 3 days), 25 to 31 days ( 6 days), and 25 to 34 days (9 days); all animals were euthanized at 38 days of age. For single-day BrdU administration after timed NTX-induced injury, mice were administered BrdU in drinking water at specific time intervals - either 24 or 48 hours (day 1 BrdU), 48-72 hours (day 2 BrdU), 72-96 hours (day 3 BrdU), 96-120 hours (day 4 BrdU), 120144 hours (day 5 BrdU), and 144-168 hours (day 6 BrdU) after injury — and animals were euthanized and tissues harvested 3 days after cessation of BrdU administration. BrdU was administered between 24 and 72 hours afterward in experiments involving IM injections of ITD-1 and NTX. 
NTX-induced injury and ITD-1 IM injection. Animals that were 24 days old were anesthetized with isoflurane, and the anterior portion of their hind limb was shaved before IM injection of $40 \mu \mathrm{L}$ of NTX $(10 \mu \mathrm{g} / \mathrm{mL}$, Latoxan, L8104) with a 28-gauge insulin syringe (BD Biosciences 329461). The needle was dipped in green tattoo dye (Harvard Apparatus, 72-9384) to track the site of muscle injury. For experiments involving IM injections of ITD-1 and NTX, $2000 \mathrm{ng}$ of ITD-1 (Tocris, 5068) was added to $30 \mu \mathrm{L}$ of NTX $(10 \mu \mathrm{g} / \mathrm{mL}$, Latoxan, L8104) and injected into the TA muscle; the contralateral TA was injected with $30 \mu \mathrm{L}$ of NTX only. At 2 and 4 days after the initial IM injection, one TA received injections of $20 \mu \mathrm{L}$ of ITD-1 (2000 ng) whereas the contralateral TA was injected with an equal volume of saline.

Muscle histology. Frozen muscles were removed from the freezer and thawed in a Leica CM1950 cryostat at $-20^{\circ} \mathrm{C}$ for $15-20$ minutes. The muscles were then sectioned at $8 \mu \mathrm{m}$ each, mounted on microscope slide, and stained for H\&E, alizarin red, and Masson's trichrome.

Microscopy. We used Olympus BX61 VS120-S5 Virtual Slide Scanning System with UPlanSApo 40×/0.95 objective, Olympus XM10 monochrome camera, and Olympus VS-ASW FL 2.7 imaging software. Analysis was performed using Olympus CellSens 1.13 and ImageJ software (National Institutes of Health). Two-photon and second harmonic images were collected on an Olympus FVMPE-RS multiphoton laser scanning microscope with a 3W Mai Tai HP Ti:Sapphire laser set at an excitation wavelength of $910 \mathrm{~nm}$. Using a 25×/1.05 NA water immersion objective, the SHG and 2-photon signal were collected using band-pass filters BP410-455 and BP495-540, respectively. Images were acquired with Olympus FV30S-SW software.

Immunofluorescence. Muscle sections were stained with anti-laminin- $\alpha 2$ (4H8-2, 1:100, Enzo), antiBrdU-biotin conjugate (B35138, 1:100, Life Technologies), DAPI nuclear stain (ProLong Gold Antifade with DAPI), anti-PDGFRa (D1E1E, 1:250, Cell Signaling Technology), p-SMAD3 (ab52903, 1:200, Abcam), and anti-PAX7 (PAX7 concentrate, 1:20, Developmental Studies Hybridoma Bank, University of Iowa). Unless indicated otherwise, muscle sections were fixed in ice-cold acetone for 10 minutes, washed in PBS (0.1\% Tween-20), and blocked for 1 hour in PBS supplemented with $10 \%$ goat serum and $10 \%$ horse serum (GeneTex), 0.1\% Tween-20 (Sigma-Aldrich), and $10 \mathrm{mg} / \mathrm{mL}$ BSA (Sigma-Aldrich). Muscle sections were incubated with primary antibodies overnight at $4^{\circ} \mathrm{C}$ and subsequently probed with Alexa Fluor secondary antibodies, including goat anti-mouse IgG1 Alexa Fluor 488/568 (A-21121/A-21124, Thermo Fisher Scientific), goat anti-rat IgG (H+L) Alexa Fluor 488/647 (A-11006/A-21247, Thermo Fisher Scientific), goat anti-rabbit IgG (H+L) Alexa Fluor 488/594 (A-11008/A-11012, Thermo Fisher Scientific), and streptavidin Alexa Fluor 488/568 (S32354/S11226, Thermo Fisher Scientific). Sections were mounted with ProLong Gold Antifade with DAPI for nuclear staining. For Pax7 immunostaining, sections were instead fixed in ice-cold 4\% PFA (Electron Microscopy Sciences, 15710), permeabilized in 0.3\% Triton X-100 in PBS, and blocked overnight with mouse on mouse blocking reagent (Vector Laboratories, MKB2213). Sections were washed in $0.05 \%$ Triton X-100 in PBS, then blocked for 30 minutes in $0.05 \%$ Triton $\mathrm{X}-100$ in PBS supplemented with $10 \%$ goat serum and $10 \mathrm{mg} / \mathrm{mL}$ fat-free milk. After blocking, sections were incubated overnight at $4^{\circ} \mathrm{C}$ in a humidified chamber with Pax7 primary antibody. Sections were then incubated with the appropriate antibodies as described above. For BrdU immunostaining, sections were fixed in ice-cold acetone for 10 minutes, incubated in $2 \mathrm{~N} \mathrm{HCl}$ at $37^{\circ} \mathrm{C}$ for 30 minutes, and briefly neutralized with $0.15 \mathrm{M}$ sodium tetraborate (Sigma-Aldrich).

TGF- $\beta 1$ ELISA. The amount of active TGF- $\beta 1$ in the triceps muscle was determined using the Quantikine ELISA mouse TGF- $\beta 1$ immunoassay (R\&D Systems, Bio-Techne, MB100B) according to the manufacturer's recommendations. Final values were normalized to total protein concentration.

Gene expression. Triceps muscles from 21- and 38-day-old B10-WT, B10-mdx, D2-WT, and D2-mdx mice were used to perform gene expression analysis. Specific mRNAs were quantified using individual TaqMan assays specific for each mRNA according to the manufacturer's protocol (Pax7, assay ID: Mm01354484 m1; myogenin, assay ID: Mm00446194_m1; and MyoD, assay ID: Mm00440387_m1). Total RNA was converted to cDNA using Random Hexamers and High Capacity cDNA Reverse Transcription Kit (Thermo Fisher Scientific, 4368814). The mRNAs were then quantified using individual TaqMan assays on an ABI QuantStudio 7 Real-Time PCR machine (Applied Biosystems) using TaqMan Fast Advanced MasterMix (Thermo Fisher Scientific, 4444556).

Isolation and in vitro osteogenesis of FAPs. FAPs were isolated from the hind limb muscles of 4-week-old B10- $m d x$ and D2- $m d x$ mice according to previously published protocols (35). Mice were euthanized and the TA, EDL, gastrocnemius, soleus, quadriceps, and psoas muscles were immediately dissected. Muscles 
were minced finely in a sterile dish and incubated in collagenase type II (2.5 U/mL, Thermo Fisher Scientific, 17101015) in PBS for 30 minutes at $37^{\circ} \mathrm{C}$. The resulting slurry was washed with sterile PBS before further digestion in Collagenase D (1.5 U/mL, Sigma-Aldrich, COLLD-RO) and Dispase II (2.4 U/mL, Sigma-Aldrich, D4693) in PBS for 60 minutes at $37^{\circ} \mathrm{C}$. Resulting slurries were passed through $100-\mu \mathrm{m}$ and $40-\mu \mathrm{m}$ strainers, and primary cells were resuspended in $1 \mathrm{~mL}$ PBS with $2 \% \mathrm{FBS}$ and $2 \mathrm{nM}$ EDTA. The primary cell suspension was stained with anti-PDGFR $\alpha$-APC $(1.0 \mu$ g per 106 cells in $100 \mu \mathrm{L}$, BioLegend, 135908) and isotype control (1.0 $\mu \mathrm{g}$ per 106 cells in $100 \mu \mathrm{L}$, BioLegend, 400512) for FACS. Cells stained with isotype control were used as a control for gating positive and negative events. Cells stained with antiPDGFR $\alpha$-APC were then sorted on an Influx cell sorter (BD, 646500) using 2-way sorting to enrich for positive PDGFR $\alpha$-expressing cells for further analysis.

Freshly isolated primary PDGFR $\alpha^{+}$population (FAPs) were plated in Matrigel-coated Nunc Lab-Tek chamber slides (Thermo Fisher Scientific, 154534) at a density of 10,000 cells/well, with 3 replicates per condition. Cells were cultured in DMEM (Lonza, 12-604F) supplemented with 20\% fetal bovine serum, $1 \%$ penicillin-streptomycin, and $2.5 \mathrm{ng} / \mathrm{mL}$ basic FGF (BioLegend, 579604) for 3 days. Osteogenic differentiation was induced by exposure to DMEM with 5\% FBS, 1\% penicillin-streptomycin, and $25 \mathrm{ng} / \mathrm{mL}$ recombinant BMP2 (R\&D Systems, Bio-Techne, 355-BM) for 3 days. Following this, cells were cultured in growth medium (DMEM with 5\% FBS and 1\% penicillin-streptomycin) for a further 4 days. Uninduced cells were not exposed to the osteogenic differentiation medium but instead cultured for 7 days in the growth medium, with the medium changed after 3 days. At the beginning of day 11, cells were fixed in chilled 4\% PFA before staining with Alkaline Phosphatase Detection Kit (Sigma-Aldrich, SCR004) to mark cells undergoing osteogenic differentiation.

Statistics. GraphPad Prism 7.03 was used for all statistical analyses of data. Statistical analysis was performed using the following: linear regression models clustered by mouse group with Tukey's post hoc multiple-comparisons adjustment, 1-way ANOVA with Tukey's post hoc comparison; 2-tailed, nonparametric Mann-Whitney $U$ test; or 2-way ANOVA, Holm-Šídák's multiple-comparisons test. The precise statistical test used varied depending on the nature of the analysis and is listed in the figure legend for each figure. Data normality was assessed for all statistical comparisons. All $P$ values less than 0.05 were considered significant. ${ }^{*} P<0.05,{ }^{* *} P<0.01,{ }^{* * *} P<0.001$, and ${ }^{* * * *} P<0.0001$. Data represent mean $\pm \mathrm{SD}$.

Study approval. All animal procedures were reviewed and approved by the Institutional Animal Care and Use Committee of Children's National Hospital in Washington, DC.

\section{Author contributions}

This study and experimental design was conceptualized by DAGM, JSN, JKJ, and TAP with contributions from YWC. Animal experiments and tissue processing were performed by DAGM, JSN, and NFH. Molecular analyses, including quantitative reverse transcription PCR and ELISA, were performed by DAGM, JSN, CBT, and NFH. Cellular analyses, including histology and immunostaining, were performed by DAGM, JSN, NFH, MN, and PA, and in vitro culture experiments were performed by MWH. Statistical analysis was performed by DAGM, JSN, and HGD. The initial manuscript was written by DAGM, JSN, JKJ, and TAP and reviewed by all authors, who all made contributions to the final manuscript. DAGM and JSN share first authorship on this manuscript, both contributing significantly to the design and execution of this work. The order of first authors was arranged according to the amount of work contributed to the manuscript and their roles in the initial development of this project.

\section{Acknowledgments}

We thank Dr. Shin'ichi Takeda of the National Center of Neurology and Psychiatry in Tokyo, Japan, for providing B6- $m d x$ mice (47). We acknowledge Shivaprasad Bhuvanendran of Children's National Hospital for imaging muscle sample using second harmonic generation. Funding for this project was obtained by DAGM, JSN, JKJ, and TAP. This work was supported by the National Institutes of Health Institute National Institute of Arthritis and Musculoskeletal and Skin Diseases (NIAMS) T32 Genetics and Genomics of Muscle Postdoctoral Training Grant (T32AR056993 to DAGM, JSN, JKJ, and TAP), Foundation to Eradicate Duchenne (to DAGM and JSN), National Institutes of Health NIAMS (R01AR055686 to JKJ), Muscular Dystrophy Association (MDA295203 to TAP, MDA480160 to JSN, MDA 477331 to MWH), Parent Project Muscular Dystrophy (to TAP), and Duchenne Parent Project - Netherlands (to JSN). Microscopy imaging was performed at the Children's National Medical Center/Children's Research Institute (CRI) 
Cellular Imaging Core, which is supported by funds from CRI and the National Institutes of Health Eunice Kennedy Shriver National Institute of Child Health and Human Development (U54HD090257 to JKJ).

Address correspondence to: Jyoti K. Jaiswal or Terence A. Partridge, 111 Michigan Avenue NW, Washington, DC, 20010-2916 USA. Phone: 202.476.6456; Email: jkjaiswal@childrensnational.org (JKJ). Phone: 44.07852259645; Email: tpartridge@childrensnational.org (TAP).

1. Hoffman EP, Brown RH, Kunkel LM. Dystrophin: the protein product of the Duchenne muscular dystrophy locus. Cell. 1987;51(6):919-928

2. Ibraghimov-Beskrovnaya O, Ervasti JM, Leveille CJ, Slaughter CA, Sernett SW, Campbell KP. Primary structure of dystrophin-associated glycoproteins linking dystrophin to the extracellular matrix. Nature. 1992;355(6362):696-702.

3. Mendell JR, et al. Evidence-based path to newborn screening for Duchenne muscular dystrophy. Ann Neurol. 2012;71(3):304-313.

4. Moens P, Baatsen PH, Maréchal G. Increased susceptibility of EDL muscles from mdx mice to damage induced by contractions with stretch. J Muscle Res Cell Motil. 1993;14(4):446-451.

5. Kharraz Y, Guerra J, Pessina P, Serrano AL, Muñoz-Cánoves P. Understanding the process of fibrosis in Duchenne muscular dystrophy. Biomed Res Int. 2014;2014:965631.

6. Shin J, Tajrishi MM, Ogura Y, Kumar A. Wasting mechanisms in muscular dystrophy. Int J Biochem Cell Biol. 2013;45(10):2266-2279.

7. Fischmann A, et al. Quantitative MRI and loss of free ambulation in Duchenne muscular dystrophy. J Neurol. 2013;260(4):969-974.

8. Wosczyna MN, Rando TA. A muscle stem cell support group: coordinated cellular responses in muscle regeneration. Dev Cell. 2018;46(2):135-143.

9. Tidball JG. Regulation of muscle growth and regeneration by the immune system. Nat Rev Immunol. 2017;17(3):165-178.

10. Vila MC, et al. Mitochondria mediate cell membrane repair and contribute to Duchenne muscular dystrophy. Cell Death Differ. 2017;24(2):330-342.

11. Duddy W, et al. Muscular dystrophy in the $\mathrm{mdx}$ mouse is a severe myopathy compounded by hypotrophy, hypertrophy and hyperplasia. Skelet Muscle. 2015;5:16.

12. Allamand V, Campbell KP. Animal models for muscular dystrophy: valuable tools for the development of therapies. Hum Mol Genet. 2000;9(16):2459-2467.

13. Fukada S, et al. Genetic background affects properties of satellite cells and mdx phenotypes. Am J Pathol. 2010;176(5):2414-2424

14. Coley WD, et al. Effect of genetic background on the dystrophic phenotype in mdx mice. Hum Mol Genet. 2016;25(1):130-145

15. van Putten M, et al. Natural disease history of the D2-mdx mouse model for Duchenne muscular dystrophy. FASEB J. 2019;33(7):8110-8124.

16. Blau HM, Webster C, Pavlath GK. Defective myoblasts identified in Duchenne muscular dystrophy. Proc Natl Acad Sci U S A. 1983;80(15):4856-4860.

17. Webster C, Blau HM. Accelerated age-related decline in replicative life-span of Duchenne muscular dystrophy myoblasts: implications for cell and gene therapy. Somat Cell Mol Genet. 1990;16(6):557-565.

18. Heydemann A, et al. Latent TGF-beta-binding protein 4 modifies muscular dystrophy in mice. J Clin Invest. 2009;119(12):3703-3712.

19. MacDonald EM, Cohn RD. TGF $\beta$ signaling: its role in fibrosis formation and myopathies. Curr Opin Rheumatol. 2012;24(6):628-634

20. Chen YW, et al. Early onset of inflammation and later involvement of TGF-beta in Duchenne muscular dystrophy. Neurology. 2005;65(6):826-834.

21. Bello L, et al. Genetic modifiers of ambulation in the Cooperative International Neuromuscular Research Group Duchenne Natural History Study. Ann Neurol. 2015;77(4):684-696.

22. Flanigan KM, et al. LTBP4 genotype predicts age of ambulatory loss in Duchenne muscular dystrophy. Ann Neurol. 2013;73(4):481-488.

23. Allen RE, Boxhorn LK. Regulation of skeletal muscle satellite cell proliferation and differentiation by transforming growth factor-beta, insulin-like growth factor I, and fibroblast growth factor. J Cell Physiol. 1989;138(2):311-315.

24. Florini JR, Roberts AB, Ewton DZ, Falen SL, Flanders KC, Sporn MB. Transforming growth factor-beta. A very potent inhibitor of myoblast differentiation, identical to the differentiation inhibitor secreted by Buffalo rat liver cells. $J$ Biol Chem 1986;261(35):16509-16513.

25. Massagué J, Cheifetz S, Endo T, Nadal-Ginard B. Type beta transforming growth factor is an inhibitor of myogenic differentiation. Proc Natl Acad Sci U S A. 1986;83(21):8206-8210.

26. Olson EN, Sternberg E, Hu JS, Spizz G, Wilcox C. Regulation of myogenic differentiation by type beta transforming growth factor. J Cell Biol. 1986;103(5):1799-1805.

27. Bernasconi P, et al. Expression of transforming growth factor-beta 1 in dystrophic patient muscles correlates with fibrosis. Pathogenetic role of a fibrogenic cytokine. J Clin Invest. 1995;96(2):1137-1144.

28. Biressi S, Miyabara EH, Gopinath SD, Carlig PM, Rando TA. A Wnt-TGFß2 axis induces a fibrogenic program in muscle stem cells from dystrophic mice. Sci Transl Med. 2014;6(267):267ra176.

29. Li Y, et al. Transforming growth factor-beta1 induces the differentiation of myogenic cells into fibrotic cells in injured skeletal muscle: a key event in muscle fibrogenesis. Am J Pathol. 2004;164(3):1007-1019.

30. Joe AW, et al. Muscle injury activates resident fibro/adipogenic progenitors that facilitate myogenesis. Nat Cell Biol. 2010;12(2):153-163.

31. Lemos DR, Paylor B, Chang C, Sampaio A, Underhill TM, Rossi FM. Functionally convergent white adipogenic progenitors of different lineages participate in a diffused system supporting tissue regeneration. Stem Cells. 2012;30(6):1152-1162.

32. Uezumi A, Fukada S, Yamamoto N, Takeda S, Tsuchida K. Mesenchymal progenitors distinct from satellite cells contribute to ectopic fat cell formation in skeletal muscle. Nat Cell Biol. 2010;12(2):143-152. 
33. Uezumi A, et al. Fibrosis and adipogenesis originate from a common mesenchymal progenitor in skeletal muscle. J Cell Sci. 2011;124(pt 21):3654-3664.

34. Lemos DR, et al. Nilotinib reduces muscle fibrosis in chronic muscle injury by promoting TNF-mediated apoptosis of fibro/ adipogenic progenitors. Nat Med. 2015;21(7):786-794.

35. Hogarth MW, et al. Fibroadipogenic progenitors are responsible for muscle loss in limb girdle muscular dystrophy $2 \mathrm{~B}$. Nat Commun. 2019;10(1):2430

36. Uezumi A, et al. Identification and characterization of PDGFR $\alpha+$ mesenchymal progenitors in human skeletal muscle. Cell Death Dis. 2014;5:e1186.

37. Lees-Shepard JB, et al. Activin-dependent signaling in fibro/adipogenic progenitors causes fibrodysplasia ossificans progressiva Nat Commun. 2018;9(1):471.

38. Willems E, et al. Small molecule-mediated TGF- $\beta$ type II receptor degradation promotes cardiomyogenesis in embryonic stem cells. Cell Stem Cell. 2012;11(2):242-252.

39. Péault B, et al. Stem and progenitor cells in skeletal muscle development, maintenance, and therapy. Mol Ther. 2007;15(5):867-877.

40. Massagué J. TGF- $\beta$ signalling in context. Nat Rev Mol Cell Biol. 2012;13(10):616-630.

41. Cohn RD, et al. Angiotensin II type 1 receptor blockade attenuates TGF-beta-induced failure of muscle regeneration in multiple myopathic states. Nat Med. 2007;13(2):204-210.

42. Girardi G, et al. TGF- $\beta$ signaling curbs cell fusion muscle regeneration [preprint]. https://doi.org/10.1101/557009. Posted on BioRxiv February 21, 2019

43. Pessina P, et al. Fibrogenic cell plasticity blunts tissue regeneration and aggravates muscular dystrophy. Stem Cell Reports. 2015;4(6):1046-1060.

44. Wosczyna MN, et al. Mesenchymal stromal cells are required for regeneration and homeostatic maintenance of skeletal muscle Cell Rep. 2019;27(7):2029-2035.e5

45. Contreras O, et al. Cross-talk between TGF- $\beta$ and PDGFR $\alpha$ signaling pathways regulates the fate of stromal fibro-adipogenic progenitors. J Cell Sci. 2019;132(19):jcs232157.

46. Mueller AA, van Velthoven CT, Fukumoto KD, Cheung TH, Rando TA. Intronic polyadenylation of PDGFR $\alpha$ in resident stem cells attenuates muscle fibrosis. Nature. 2016;540(7632):276-279.

47. Araki E, et al. Targeted disruption of exon 52 in the mouse dystrophin gene induced muscle degeneration similar to that observed in Duchenne muscular dystrophy. Biochem Biophys Res Commun. 1997;238(2):492-497.

48. Rosenblatt JD, Lunt AI, Parry DJ, Partridge TA. Culturing satellite cells from living single muscle fiber explants. In Vitro Cell Dev Biol Anim. 1995;31(10):773-779.

49. Duddy WJ, Cohen T, Duguez S, Partridge TA. The isolated muscle fibre as a model of disuse atrophy: characterization using PhAct, a method to quantify f-actin. Exp Cell Res. 2011;317(14):1979-1993.

50. Novak JS, et al. Myoblasts and macrophages are required for therapeutic morpholino antisense oligonucleotide delivery to dystrophic muscle. Nat Commun. 2017;8(1):941. 